\title{
Classical Liouville three-point functions from Riemann-Hilbert analysis
}

\author{
Daigo Honda and Shota Komatsu \\ Institute of Physics, University of Tokyo, \\ Komaba, Meguro-ku, Tokyo 153-8902, Japan \\ E-mail: dhonda@hep1.c.u-tokyo.ac.jp, skomatsu@hep1.c.u-tokyo.ac.jp
}

ABStRACT: We study semiclassical correlation functions in Liouville field theory on a two-sphere when all operators have large conformal dimensions. In the usual approach, such computation involves solving the classical Liouville equation, which is known to be extremely difficult for higher-point functions. To overcome this difficulty, we propose a new method based on the Riemann-Hilbert analysis, which is applied recently to the holographic calculation of correlation functions in AdS/CFT. The method allows us to directly compute the correlation functions without solving the Liouville equation explicitly. To demonstrate its utility, we apply it to three-point functions, which are known to be solvable, and confirm that it correctly reproduces the classical limit of the DOZZ formula for quantum three-point functions. This provides good evidence for the validity of this method.

Keywords: Field Theories in Lower Dimensions, Nonperturbative Effects, Integrable Field Theories, Conformal and W Symmetry

ARXIV EPRINT: 1311.2888 


\section{Contents}

1 Introduction 1

2 Classical Liouville correlation functions $\quad 2$

2.1 Semiclassical analysis of Liouville field theory 3

2.2 Correlation functions and Wronskians 5

3 Determination of Wronskians $\quad 8$

3.1 Monodromy relation 8

$\begin{array}{ll}3.2 \text { Poles of Wronskians } & 10\end{array}$

3.3 Zeros of Wronskians 11

$\begin{array}{lll}\text { 3.3.1 WKB approximation and WKB curves } & 12\end{array}$

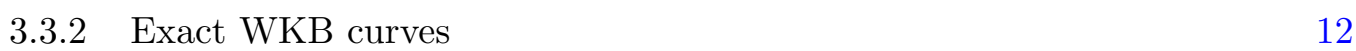

$\begin{array}{lll}\text { 3.3.3 Determination of zeros } & 17\end{array}$

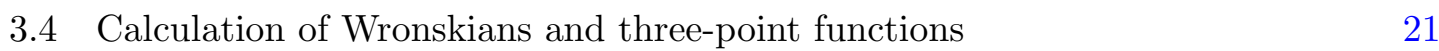

4 Discussion and future direction $\quad 25$

\section{Introduction}

Liouville field theory is one of a few well-studied irrational conformal field theories and has been studied thoroughly in various context such as noncritical string theory, twodimensional quantum gravity, tachyon condensation and quantum gravity in the de-Sitter spacetime (see comprehensive reviews [1,2] and references therein). Furthermore, it has been drawing renewed attention recently because of its relation to four-dimensional $\mathcal{N}=2$ supersymmetric gauge theories, known as the AGT correspondence [3].

In this paper, we consider the semiclassical limit of the Liouville field theory, in which the central charge goes to positive infinity. In particular, we discuss semiclassical correlation functions on a two-sphere when all vertex operators have large conformal dimensions. In the AGT correspondence, the semiclassical limit of the Liouville field theory is known to correspond to a special limit of the gauge theory, called the Nekrasov-Shatashvilli limit [4]. In this limit, an intriguing relation between the gauge theories and the quantum integrable models has been discovered [4-23]. Although this limit is extensively studied in the literature, the nature and the mechanism of this mysterious relation are still to be elucidated. Thus, for deeper understanding of this relation, it would be worthwhile to investigate the structure of the semiclassical Liouville theory more in detail.

To compute the semiclassical correlation functions in the usual method, we need the explicit form of the solution to the classical Liouville equation. However, the solution to the Liouville equation is known only for three-point functions [24-26], and it is considered 
to be extremely difficult to obtain the solutions for higher-point functions. ${ }^{1}$ To overcome this difficulty, we propose a new method, which does not necessitate the explicit solution to the Liouville equation and is based on a kind of Riemann-Hilbert method developed recently for the holographic calculation of correlation functions in AdS/CFT [28-32]. To demonstrate its utility, we study the three-point functions in this paper and show that it reproduces the known results correctly.

The key of the method is a certain second-order ordinary differential equation ${ }^{2}$ associ- $^{-}$ ated with the solution to the classical Liouville equation. This equation plays a pivotal role in the analysis since the classical action of the Liouville field theory can be re-expressed in terms of the Wronskians of this differential equation. To compute such Wronskians, we take the following steps. First, from the simple fact that there is no insertion of vertex operators at infinity, we determine certain products of the Wronskians. Next, to compute the individual Wronskians, not just their products, we introduce an extra parameter called spectral parameter and consider a one-parameter deformation of the Wronskians. Then, we discuss the analyticity of the Wronskians with respect to the spectral parameter using a newly introduced quantity called exact WKB curves, which is a generalization of the ordinary WKB curves defined in [34]. With the exact WKB curves, we can fully determine the analyticity of the Wronskians. Then, using the analytic properties thus obtained, we set up the Riemann-Hilbert problem and solve it explicitly in terms of gamma functions. The three-point functions computed in this way turn out to agree completely with the classical limit of the DOZZ three-point functions [24, 35, 36]. The agreement provides strong evidence for the validity and the utility of this method.

The rest of this paper is organized as follows. Firstly, in section 2, we will give a brief summary on the semiclassical limit of the Liouville correlation functions. In particular, we explain that the classical action can be expressed in terms of the Wronskians of certain ordinary differential equations. Secondly, in section 3, we will determine the Wronskians using the exact WKB curves and the Riemann-Hilbert analysis, and compute the threepoint functions exactly. Finally, in section 4, we will conclude and indicate several future directions including the generalization to the higher-point functions and the relation to the four-dimensional $\mathcal{N}=2$ supersymmetric gauge theories.

\section{Classical Liouville correlation functions}

In this section, we summarize the basics of semiclassical analysis of the Liouville field theory. First, starting from the fully quantum path integral expression of the Liouville field theory, we show that the semiclassical approximation is valid when vertex operators have large conformal dimensions. Then, we move onto the three-point functions. The semiclassical limit of the three-point functions is a well-studied subject (see [24-26]). However,

\footnotetext{
${ }^{1}$ Although one can write down the general form of the solutions to the Liouville equation in terms of a meromorphic function and its complex conjugate (see for instance [27]), it is difficult to determine their explicit form in the case of higher-point functions.

${ }^{2}$ This differential equation originates from the null-vector decoupling equation in the quantum Liouville field theory and is often referred to as the oper in the literature [33].
} 
our approach is different from the previous works as we do not make use of the classical solutions. After reviewing the long-known fact that the classical solutions of the Liouville equation can be constructed from the solutions of a certain second-order ordinary differential equation, we show that the semiclassical correlation functions can be re-expressed in terms of the Wronskians of this differential equation. Such Wronskians will be directly evaluated in the next section.

\subsection{Semiclassical analysis of Liouville field theory}

The path integral expression of the Liouville correlation function on a two-sphere is given as follows ${ }^{3}$

$$
\left\langle\mathcal{V}_{1}\left(z_{1}\right) \mathcal{V}_{2}\left(z_{2}\right) \cdots \mathcal{V}_{n}\left(z_{n}\right)\right\rangle=\int \mathcal{D} \phi \exp \left[-\frac{1}{2 \pi b^{2}} \int d^{2} z\left(\partial \phi \bar{\partial} \phi+2 \lambda e^{2 \phi}\right)\right] \prod_{i=1}^{n} \mathcal{V}_{i}\left(z_{i}\right)
$$

where $\mathcal{V}_{i}$ is a vertex operator given by

$$
\mathcal{V}_{i}\left(z_{i}\right)=e^{2 \alpha_{i} \phi\left(z_{i}\right) / b}
$$

Precisely speaking, the boundary term at infinity is necessary to make the action integral convergent [24-26]. However, in what follows, we will not evaluate the action directly and the precise form of the boundary term will not be needed.

Let us now discuss the semiclassical limit. The semiclassical limit of the Liouville field theory is basically $b \rightarrow 0$ limit. As (2.1) shows, the action part has $1 / b^{2}$ prefactor, which diverges in the limit. Therefore, the path integral in this limit is dominated by its saddlepoint value. However, if we take the naive $b \rightarrow 0$ limit with $\alpha_{i}$ fixed, the vertex operators in the limit scale as $1 / b$, which is $b(\rightarrow 0)$ times smaller than the action part. Then, the saddle point will be insensitive to the vertex operators and the result will become trivial. ${ }^{4}$ To obtain a nontrivial result, we need to properly scale $\alpha_{i}$ so that $\eta_{i} \equiv b \alpha_{i}$ stays finite. We call the operators whose conformal dimensions are scaled in this way "heavy" operators. In such a limit, the path integral (2.1) can be evaluated as

$$
\begin{aligned}
& \left\langle\mathcal{V}_{1}\left(z_{1}\right) \mathcal{V}_{2}\left(z_{2}\right) \cdots \mathcal{V}_{n}\left(z_{n}\right)\right\rangle \stackrel{b \rightarrow 0}{\sim} \exp \left(-\frac{S}{b^{2}}\right), \\
& S \equiv \frac{1}{2 \pi} \int d^{2} z\left(\partial \phi_{*} \bar{\partial} \phi_{*}+2 \lambda e^{2 \phi_{*}}-4 \pi \sum_{i} \eta_{i} \phi_{*} \delta^{2}\left(z-z_{i}\right)\right),
\end{aligned}
$$

where $\phi_{*}$ is the saddle-point value of $\phi$ which satisfies the classical Liouville equation,

$$
\partial \bar{\partial} \phi_{*}=\lambda e^{2 \phi_{*}}-\pi \sum_{i} \eta_{i} \delta^{2}\left(z-z_{i}\right)
$$

In what follows, we only consider the case where $\eta_{i}$, which parametrize the vertex operators, are real-valued and smaller than $1 / 2$. When $\eta_{i}$ satisfy these conditions, the exponential

\footnotetext{
${ }^{3}$ This action, which is slightly different from the usual one, is obtained after the field redefinition $\phi \rightarrow \phi / b$. For a further detail, see, for instance, [26].

${ }^{4}$ In such cases, the quantum correction (or equivalently the one-loop correction) will become important.
} 
term in (2.5) can be neglected ${ }^{5}$ in the vicinity of the vertex operators and the asymptotic behavior of $\phi_{*}$ is given by

$$
\phi_{*} \stackrel{z \rightarrow z_{i}}{\sim}-2 \eta_{i} \ln \left|z-z_{i}\right|+C_{i}+O\left(z-z_{i}\right) .
$$

The inequality $\eta_{i}<1 / 2$ is known as the Seiberg bound [27] in the literature. The modern interpretation ${ }^{6}$ of this bound is that, in the quantum Liouville field theory, the operator with $\eta>1 / 2$ is equivalent to the operator with $1-\eta$ by some rescaling factor, called the reflection coefficient.

There is another constraint we impose on the values of $\eta_{i}$. Integrating (2.5) over the two-sphere and using the Gauss-Bonnet theorem, we obtain

$$
\sum_{i} \eta_{i}-1=\frac{\lambda}{\pi}(\text { Area })
$$

where (Area) denotes the area of the Riemann surface computed with the metric $g_{i j}=$ $e^{\phi_{*}} \delta_{i j}$. Therefore, when the Liouville equation has a real-valued solution, (2.7) must be positive. This provides the necessary condition for the existence of the real solution, which we impose throughout this paper. In summary, we require the parameters $\eta_{i}$ to be in the following region, which we call the physical region,

$$
\eta_{i}<\frac{1}{2}, \quad \sum_{i} \eta_{i}>1
$$

Note that, for the three-point functions, $\eta_{i}>0$ follows from the above conditions.

Now, using (2.5), we can define the following holomorphic quantity: ${ }^{7}$

$$
T(z) \equiv-\left(\partial \phi_{*}\right)^{2}+\partial^{2} \phi_{*} .
$$

This quantity is, in fact, a semiclassical limit of the stress-energy tensor and plays an important role in the subsequent analysis. From (2.6), the asymptotic behavior of $T(z)$ can be determined in the following form:

$$
T(z)=\sum_{i} \frac{\eta_{i}\left(1-\eta_{i}\right)}{\left(z-z_{i}\right)^{2}}+\frac{a_{i}}{z-z_{i}} .
$$

Here, the parameters $a_{i}$ are called accessory parameters. They are constrained by the condition that the stress-energy tensor is not singular and decays as $T(z) \sim z^{-4}$ at infinity in the following way:

$$
\sum_{i} a_{i}=0, \sum_{i}\left(a_{i} z_{i}+\eta_{i}\left(1-\eta_{i}\right)\right)=0, \sum_{i}\left(a_{i} z_{i}^{2}+2 \eta_{i}\left(1-\eta_{i}\right) z_{i}\right)=0 .
$$

\footnotetext{
${ }^{5}$ If we consider the operator with $\eta>1 / 2$, the asymptotic behavior will be affected by the exponential term in (2.5).

${ }^{6}$ In the context of two-dimensional gravity, the Liouville field plays the role of the metric $g_{i j}=e^{2 \phi} \delta_{i j}$ on the two-sphere in the conformal gauge. Then, the insertion point of a vertex operator with $\eta$ becomes a singularity with a deficit angle $2 \pi(1-2 \eta)$ and the Seiberg bound $\eta<1 / 2$ naturally follows from the geometric requirement.

${ }^{7}$ We can also define the anti-holomorphic quantity which corresponds to the anti-chiral stress energy tensor by $\bar{T}(\bar{z}) \equiv-\left(\bar{\partial} \phi_{*}\right)^{2}+\bar{\partial}^{2} \phi_{*}$.
} 
In the case of the three-point functions, (2.11) is restrictive enough to fully determine the form of $T(z)$ as

$$
T(z)=\left(\frac{\eta_{1}\left(1-\eta_{1}\right) z_{12} z_{13}}{z-z_{1}}+\frac{\eta_{2}\left(1-\eta_{2}\right) z_{21} z_{23}}{z-z_{2}}+\frac{\eta_{3}\left(1-\eta_{3}\right) z_{31} z_{32}}{z-z_{3}}\right) \frac{1}{\left(z-z_{1}\right)\left(z-z_{2}\right)\left(z-z_{3}\right)},
$$

where $z_{i j}$ is given by $z_{i}-z_{j}$.

Let us now explain how to compute the semiclassical correlation functions. The most straightforward way to compute them is to evaluate the integrand of the path integral (2.1), which consist of the action and the vertex operators, on the saddle-point classical solution. Although this line of approach was taken in the study of correlation functions in AdS/CFT, there is much an easier way for the Liouville field theory. The idea is to consider the variation of the semiclassical correlation functions with respect to the parameters of the vertex operators, $\eta_{i}$. The correlation functions evaluated on the saddle-point depends on $\eta_{i}$ in two different ways: first, the integrand has an explicit dependence on $\eta_{i}, \exp \left(2 \eta_{i} \phi / b^{2}\right)$. Second, as the saddle-point solution itself depends on $\eta_{i}$, the integrand depends on $\eta_{i}$ implicitly through the saddle-point solution. However, the second dependence is always of the form,

$$
\frac{\delta S}{\delta \phi} \frac{\delta \phi}{\delta \eta_{i}} \delta \eta_{i}
$$

which vanishes since the saddle point satisfies the equation of motion. Therefore, we conclude the only dependence we need to consider is the explicit dependence on $\eta_{i}$ in the integrand. This way, we arrive at the following important formula:

$$
\frac{\partial S}{\partial \eta_{i}}=-2 C_{i}
$$

where $C_{i}$ is the subleading term ${ }^{8}$ in (2.6). Consequently, the evaluation of the semiclassical limit of the correlation functions boils down to the evaluation of $C_{i}$.

\subsection{Correlation functions and Wronskians}

In the previous works, $C_{i}$ is determined by using the explicit solutions of the classical Liouville equation [24-26]. However, it is extremely difficult to obtain the explicit solutions for the higher-point functions. To overcome this problem, we will propose another method based on the Riemann-Hilbert analysis in this paper. The purpose of this section is to express $C_{i}$ in (2.14) in a form to which our method is readily applicable.

First, we use the following famous fact of the classical Liouville field theory: the classical solution of the Liouville field theory can be expressed as the bilinear of the solutions to the ordinary differential equation,

$$
\left(\partial^{2}+T(z)\right) \psi_{i}=0, \quad\left(\bar{\partial}^{2}+\bar{T}(\bar{z})\right) \bar{\psi}_{i}=0,
$$

\footnotetext{
${ }^{8}$ Although the leading term in (2.6) produces the divergent contribution, it is absorbed by the renormalization of the vertex operators. For details, see [24].
} 
as follows:

$$
\begin{aligned}
& e^{-\phi}=\sqrt{\lambda}\left(\psi_{1} \bar{\psi}_{1}-\psi_{2} \bar{\psi}_{2}\right), \\
& \left\langle\psi_{1}, \psi_{2}\right\rangle\left\langle\bar{\psi}_{1}, \bar{\psi}_{2}\right\rangle=1,
\end{aligned}
$$

where $\langle A, B\rangle$ is a Wronskian between $A$ and $B$, defined by $A \partial B-B \partial A$ or $A \bar{\partial} B-B \bar{\partial} A$. Existence of such a linear differential equation is one of the important characteristics of integrable systems and plays a pivotal role in the context of the holographic calculation of correlation functions in AdS/CFT [28-32]. Following the convention in that context, we will call the equation (2.15) the auxiliary linear problem.

Let us now make an important remark on (2.16). Although it is always true that the classical solution of the Liouville field theory can be represented by a certain bilinear of the solutions to the auxiliary linear problem, not all the bilinears provide a consistent solution of the Liouville field theory. This is because, while the left hand side of (2.16), $e^{-\phi}$, is always single-valued, the single-valuedness is not guaranteed if we choose a bilinear arbitrarily. Thus, the correct statement is as follows: If the bilinear of the auxiliary linear problem, defined by the right hand side of (2.16), is single-valued on the Riemann surface we consider, it provides a classical solution of the Liouville field theory.

Having clarified the relation between the solution of the Liouville field theory and the solutions to the auxiliary linear problem, let us discuss the asymptotic property near the vertex operators. In the vicinity of the vertex operator, $z_{i}$, there are two independent solutions $i_{ \pm}$to the auxiliary linear problem (2.15) with different asymptotic behavior.

$$
\begin{array}{ll}
i_{+} \sim\left(z-z_{i}\right)^{\eta_{i}}, & i_{-} \sim\left(z-z_{i}\right)^{1-\eta_{i}} /\left(1-2 \eta_{i}\right), \\
\bar{i}_{+} \sim\left(\bar{z}-\bar{z}_{i}\right)^{\eta_{i}}, & \bar{i}_{-} \sim\left(\bar{z}-\bar{z}_{i}\right)^{1-\eta_{i}} /\left(1-2 \eta_{i}\right) .
\end{array}
$$

Here, we normalized $i_{ \pm}$so that they satisfy the normalization conditions, $\left\langle i_{+}, i_{-}\right\rangle=$ $\left\langle\bar{i}_{+}, \bar{i}_{-}\right\rangle=1$. In terms of $i_{ \pm}$and $\bar{i}_{ \pm}$defined above, the solutions to (2.15) which appears in the formula (2.16) can be expressed as follows.

$$
\begin{aligned}
& \psi_{k}=\left\langle\psi_{k}, i_{-}\right\rangle i_{+}-\left\langle\psi_{k}, i_{+}\right\rangle i_{-}, \\
& \bar{\psi}_{k}=\left\langle\bar{\psi}_{k}, \bar{i}_{-}\right\rangle \bar{i}_{+}-\left\langle\bar{\psi}_{k}, \bar{i}_{+}\right\rangle \bar{i}_{-},
\end{aligned}
$$

These expressions are useful to determine the asymptotic behavior of $\psi_{k}$ and $\bar{\psi}_{k}$. Since $\eta_{i}$ is smaller ${ }^{9}$ than $1-\eta_{i}, \psi_{k}$ and $\bar{\psi}_{k}$ can be approximated near $z_{i}$ as

$$
\psi_{k} \sim\left\langle\psi_{k}, i_{-}\right\rangle\left(z-z_{i}\right)^{\eta_{i}}, \quad \bar{\psi}_{k} \sim\left\langle\bar{\psi}_{k}, \bar{i}_{-}\right\rangle\left(\bar{z}-\bar{z}_{i}\right)^{\eta_{i}} .
$$

Thus, the asymptotic behavior of $\phi$ can be determined as

$$
\phi \sim-2 \eta_{i} \log \left|z-z_{i}\right|-\log \left(\left\langle\psi_{1}, i_{-}\right\rangle\left\langle\bar{\psi}_{1}, \bar{i}_{-}\right\rangle-\left\langle\psi_{2}, i_{-}\right\rangle\left\langle\bar{\psi}_{2}, \bar{i}_{-}\right\rangle\right)-\frac{1}{2} \log \lambda .
$$

We have not hitherto considered seriously the single-valuedness of $e^{-\phi}$. Thus, let us next discuss what kind of constraints the single-valuedness imposes. Although we will

\footnotetext{
${ }^{9}$ Recall that $\eta_{i}$ is smaller than $1 / 2$ owing to the condition (2.8).
} 
henceforth consider only the three-point functions, the discussion in this subsection can be generalized to the higher-point functions. First, consider the single-valuedness around $z_{1}$. To be single-valued, or equivalently monodromy-free, around $z_{1}, e^{-\phi}$ must be of the following form:

$$
e^{-\phi} / \sqrt{\lambda}=A 1_{+} \overline{1}_{+}-A^{-1} 1_{-} \overline{1}_{-} .
$$

In other words, $\psi_{1}$ and $\bar{\psi}_{1}$ must be proportional to $1_{+}$and $\overline{1}_{+}$, and $\psi_{2}$ and $\bar{\psi}_{2}$ must be proportional to $1_{-}$and $\overline{1}_{-}$. For the reality of $e^{-\phi}$, we should take $A$ to be real. Now, let us re-express (2.24) in terms of the solution around $z_{2} \cdot{ }^{10}$ Using the following relation,

$$
1_{+}=\left\langle 1_{+}, 2_{-}\right\rangle 2_{+}-\left\langle 1_{+}, 2_{+}\right\rangle 2_{-} \text {etc. }
$$

we obtain

$$
\begin{aligned}
e^{-\phi} / \sqrt{\lambda}= & \left(A\left\langle 1_{+}, 2_{-}\right\rangle\left\langle\overline{1}_{+}, \overline{2}_{-}\right\rangle-A^{-1}\left\langle 1_{-}, 2_{-}\right\rangle\left\langle\overline{1}_{-}, \overline{2}_{-}\right\rangle\right) 2_{+} \overline{2}_{+} \\
& +\left(A\left\langle 1_{+}, 2_{+}\right\rangle\left\langle\overline{1}_{+}, \overline{2}_{+}\right\rangle-A^{-1}\left\langle 1_{-}, 2_{+}\right\rangle\left\langle\overline{1}_{-}, \overline{2}_{+}\right\rangle\right) 2_{-} \overline{2}_{-} \\
& -\left(A\left\langle 1_{+}, 2_{-}\right\rangle\left\langle\overline{1}_{+}, \overline{2}_{+}\right\rangle-A^{-1}\left\langle 1_{-}, 2_{-}\right\rangle\left\langle\overline{1}_{-}, \overline{2}_{+}\right\rangle\right) 2_{+} \overline{2}_{-} \\
& -\left(A\left\langle 1_{+}, 2_{+}\right\rangle\left\langle\overline{1}_{+}, \overline{2}_{-}\right\rangle-A^{-1}\left\langle 1_{-}, 2_{+}\right\rangle\left\langle\overline{1}_{-}, \overline{2}_{-}\right\rangle\right) 2_{-} \overline{2}_{+} .
\end{aligned}
$$

$e^{-\phi}$ is single-valued if and only if the last two terms in the above equation vanishes. Therefore, we obtain the following constraints,

$$
\begin{aligned}
& A\left\langle 1_{+}, 2_{-}\right\rangle\left\langle\overline{1}_{+}, \overline{2}_{+}\right\rangle-A^{-1}\left\langle 1_{-}, 2_{-}\right\rangle\left\langle\overline{1}_{-}, \overline{2}_{+}\right\rangle=0, \\
& A\left\langle 1_{+}, 2_{+}\right\rangle\left\langle\overline{1}_{+}, \overline{2}_{-}\right\rangle-A^{-1}\left\langle 1_{-}, 2_{+}\right\rangle\left\langle\overline{1}_{-}, \overline{2}_{-}\right\rangle=0 .
\end{aligned}
$$

Solving the above two equations (2.27) and (2.28), we arrive at the following expression:

$$
\frac{e^{-\phi}}{\sqrt{\lambda}}=\sqrt{\left|\frac{\left\langle 1_{-}, 2_{-}\right\rangle\left\langle 1_{-}, 2_{+}\right\rangle}{\left\langle 1_{+}, 2_{+}\right\rangle\left\langle 1_{+}, 2_{-}\right\rangle}\right|} 1_{+} \overline{1}_{+}-\sqrt{\left|\frac{\left\langle 1_{+}, 2_{+}\right\rangle\left\langle 1_{+}, 2_{-}\right\rangle}{\left\langle 1_{-}, 2_{-}\right\rangle\left\langle 1_{-}, 2_{+}\right\rangle}\right|} 1_{-} \overline{1}_{-} .
$$

From the single-valuedness condition around $z_{3}$, we can derive an equation similar to (2.29), but with $2_{ \pm}$replaced with $3_{ \pm}$. Combining these two expressions, the following symmetric expression can be written down:

$$
\begin{aligned}
\frac{e^{-\phi}}{\sqrt{\lambda}}= & \left|\frac{\left\langle 1_{-}, 2_{-}\right\rangle\left\langle 1_{-}, 2_{+}\right\rangle}{\left\langle 1_{+}, 2_{+}\right\rangle\left\langle 1_{+}, 2_{-}\right\rangle} \frac{\left\langle 3_{-}, 1_{-}\right\rangle\left\langle 3_{+}, 1_{-}\right\rangle}{\left\langle 3_{+}, 1_{+}\right\rangle\left\langle 3_{-}, 1_{+}\right\rangle}\right|^{\frac{1}{4}} 1_{+} \overline{1}_{+} \\
& -\left|\frac{\left\langle 1_{+}, 2_{+}\right\rangle\left\langle 1_{+}, 2_{-}\right\rangle}{\left\langle 1_{-}, 2_{-}\right\rangle\left\langle 1_{-}, 2_{+}\right\rangle} \frac{\left\langle 3_{+}, 1_{+}\right\rangle\left\langle 3_{-}, 1_{+}\right\rangle}{\left\langle 3_{-}, 1_{-}\right\rangle\left\langle 3_{+}, 1_{-}\right\rangle}\right|^{\frac{1}{4}} 1_{-} \overline{1}_{-} .
\end{aligned}
$$

\footnotetext{
${ }^{10}$ The monodromy-free condition around $z_{3}$ is trivial since the contour around $z_{3}$ can be decomposed into a sum of contours around $z_{1}$ and $z_{2}$.
} 
The expression in terms of $2_{ \pm}$or in terms of $3_{ \pm}$can be obtained by the permutation of 1 , 2 and 3 as follows:

$$
\begin{aligned}
& \frac{e^{-\phi}}{\sqrt{\lambda}}=\left|\frac{\left\langle 2_{-}, 3_{-}\right\rangle\left\langle 2_{-}, 3_{+}\right\rangle}{\left\langle 2_{+}, 3_{+}\right\rangle\left\langle 2_{+}, 3_{-}\right\rangle} \frac{\left\langle 1_{-}, 2_{-}\right\rangle\left\langle 1_{+}, 2_{-}\right\rangle}{\left\langle 1_{+}, 2_{+}\right\rangle\left\langle 1_{-}, 2_{+}\right\rangle}\right|^{\frac{1}{4}} 2_{+} \overline{2}_{+} \\
&-\left|\frac{\left\langle 2_{+}, 3_{+}\right\rangle\left\langle 2_{+}, 3_{-}\right\rangle}{\left\langle 2_{-}, 3_{-}\right\rangle\left\langle 2_{-}, 3_{+}\right\rangle} \frac{\left\langle 1_{+}, 2_{+}\right\rangle\left\langle 1_{-}, 2_{+}\right\rangle}{\left\langle 1_{-}, 2_{-}\right\rangle\left\langle 1_{+}, 2_{-}\right\rangle}\right|^{\frac{1}{4}} 2_{-} \overline{2}_{-}, \\
& \frac{e^{-\phi}}{\sqrt{\lambda}}=\left|\frac{\left\langle 3_{-}, 1_{-}\right\rangle\left\langle 3_{-}, 1_{+}\right\rangle}{\left\langle 3_{+}, 1_{+}\right\rangle\left\langle 3_{+}, 1_{-}\right\rangle} \frac{\left\langle 2_{-}, 3_{-}\right\rangle\left\langle 2_{+}, 3_{-}\right\rangle}{\left\langle 2_{+}, 3_{+}\right\rangle\left\langle 2_{-}, 3_{+}\right\rangle}\right|^{\frac{1}{4}} 3_{+} \overline{3}_{+} \\
&-\left|\frac{\left\langle 3_{+}, 1_{+}\right\rangle\left\langle 3_{+}, 1_{-}\right\rangle}{\left\langle 3_{-}, 1_{-}\right\rangle\left\langle 3_{-}, 1_{+}\right\rangle} \frac{\left\langle 2_{+}, 3_{+}\right\rangle\left\langle 2_{-}, 3_{+}\right\rangle}{\left\langle 2_{-}, 3_{-}\right\rangle\left\langle 2_{+}, 3_{-}\right\rangle}\right|^{\frac{1}{4}} 3_{-} \overline{3}_{-} .
\end{aligned}
$$

From (2.30)-(2.32), the parameters $C_{i}$ can be easily read off as

$$
C_{i}=-\frac{1}{2} \log \lambda+\frac{1}{4}\left(\sum_{\epsilon= \pm, j \neq i} \log \left|\left\langle i_{+}, j_{\epsilon}\right\rangle\right|-\sum_{\epsilon= \pm, j \neq i} \log \left|\left\langle i_{-}, j_{\epsilon}\right\rangle\right|\right) .
$$

Thus, the semiclassical three-point functions can be computed once we know the values of the Wronskians $\left\langle i_{ \pm}, j_{ \pm}\right\rangle$.

\section{Determination of Wronskians}

Having seen that the Wronskians are fundamental objects for the evaluation of the semiclassical three-point functions, we shall evaluate such Wronskians in this section. First we show that certain products of Wronskians can be determined from the triviality of the monodromy at infinity. To obtain the individual Wronskians, we next consider a one-parameter deformation of the auxiliary linear problem and the Wronskians. Then, we determine the analyticity of the Wronskians and set up the Riemann-Hilbert problem. Finally, solving the Riemann-Hilbert problem, we compute the parameters $C_{i}$ in $(2.33)$ and see that $C_{i}$ 's thus computed coincide with the known result derived in [24-26].

\subsection{Monodromy relation}

Let us consider the monodromy of solutions to the first equation of (2.15). Since we are considering the solutions on the sphere, only noncontractable cycles are cycles around punctures (vertex operators). The eigenvalues of each monodromy matrix are determined purely by the local behavior of the solutions and therefore can be expressed purely by the parameter of the corresponding vertex operator.

$$
\Omega_{i} \sim\left(\begin{array}{cc}
e^{i p_{i}} & 0 \\
0 & e^{-i p_{i}}
\end{array}\right)
$$

where $p_{i}$ is the Liouville momentum defined by $p_{i} \equiv 2 \pi \eta_{i}$.

Although $\Omega_{i}$ 's can be separately diagonalized as (3.1), they cannot be diagonalized simultaneously, owing to the nontrivial global behavior of the solution to the auxiliary linear 
problem. Nevertheless, the simple relation, $\Omega_{1} \Omega_{2} \Omega_{3}=1$, which expresses the triviality of the monodromy at infinity, is enough to restrict the form of $\Omega_{i}$ sufficiently, although not completely. To see this, let us take a basis of solutions in which $\Omega_{1}$ is diagonal,

$$
\Omega_{1}=\left(\begin{array}{cc}
e^{i p_{1}} & 0 \\
0 & e^{-i p_{1}}
\end{array}\right),
$$

and express the matrix $\Omega_{2}$ in this basis as

$$
\Omega_{2}=\left(\begin{array}{ll}
a & b \\
c & d
\end{array}\right) .
$$

Since $\Omega_{2}$ has eigenvalues $\exp \left( \pm i p_{2}\right), a-d$ must satisfy the following relations.

$$
a+d=2 \cos p_{2}, \quad a d-b c=1 .
$$

Next, let us consider $\Omega_{3}$. Owing to the relation, $\Omega_{1} \Omega_{2} \Omega_{3}=1, \Omega_{3}$ can be expressed in terms of $a$ - $d$ as follows.

$$
\Omega_{3}=\Omega_{2}^{-1} \Omega_{1}^{-1}=\left(\begin{array}{cc}
e^{-i p_{1}} d & -e^{i p_{1}} b \\
-e^{-i p_{1}} c & e^{i p_{1}} a
\end{array}\right) .
$$

Then, using the fact $\Omega_{3}$ has eigenvalues $\exp \left( \pm i p_{3}\right)$, we can obtain one more constraint.

$$
e^{-i p_{1}} d+e^{i p_{1}} a=2 \cos p_{3}
$$

It is easy to see that (3.4) and (3.6) completely determines $a$ and $d$, while only the product $b c$ can be determined. ${ }^{11}$ With these explicit form of the monodromy matrix, we can compute various Wronskians between $i_{ \pm}$, which are the eigenvectors of the monodromy matrices. However, because of the aforementioned ambiguity of $b$ and $c$, only the following products of Wronskians can be determined:

$$
\begin{aligned}
\left\langle 1_{+}, 2_{+}\right\rangle\left\langle 1_{-}, 2_{-}\right\rangle= & \frac{\sin \frac{p_{1}+p_{2}-p_{3}}{2} \sin \frac{p_{1}+p_{2}+p_{3}}{2}}{\sin p_{1} \sin p_{2}}, \\
\left\langle 2_{+}, 3_{+}\right\rangle\left\langle 2_{-}, 3_{-}\right\rangle= & \frac{\sin \frac{-p_{1}+p_{2}+p_{3}}{2} \sin \frac{p_{1}+p_{2}+p_{3}}{2}}{\sin p_{2} \sin p_{3}}, \\
\left\langle 3_{+}, 1_{+}\right\rangle\left\langle 3_{-}, 1_{-}\right\rangle & =\frac{\sin \frac{p_{1}-p_{2}+p_{3}}{2} \sin \frac{p_{1}+p_{2}+p_{3}}{2}}{\sin p_{3} \sin p_{1}}, \\
\left\langle 1_{+}, 2_{-}\right\rangle\left\langle 1_{-}, 2_{+}\right\rangle & =\frac{\sin \frac{p_{1}-p_{2}-p_{3}}{2} \sin \frac{p_{1}-p_{2}+p_{3}}{2}}{\sin p_{1} \sin p_{2}}, \\
\left\langle 2_{+}, 3_{-}\right\rangle\left\langle 2_{-}, 3_{+}\right\rangle & =\frac{\sin \frac{-p_{1}+p_{2}-p_{3}}{2} \sin \frac{p_{1}+p_{2}-p_{3}}{2}}{\sin p_{2} \sin p_{3}}, \\
\left\langle 3_{+}, 1_{-}\right\rangle\left\langle 3_{-}, 1_{+}\right\rangle= & \frac{\sin \frac{-p_{1}-p_{2}+p_{3}}{2} \sin \frac{-p_{1}+p_{2}+p_{3}}{2}}{\sin p_{3} \sin p_{1}} .
\end{aligned}
$$

\footnotetext{
${ }^{11}$ For a detail of the derivation, see [29].
} 
To compute the semiclassical three-point functions, we need to compute the individual Wronskians, not just their products. For this purpose, let us introduce the following oneparameter deformation of the auxiliary linear problem:

$$
\left(\partial^{2}+\frac{1}{\xi^{2}} T(z)\right) \psi=0 .
$$

In what follows, we will call $\xi$ the spectral parameter following the convention in integrable systems. (3.13) coincide in form with the Schrödinger equation ${ }^{12}$ if we identify $\xi$ with $\hbar$. The asymptotic behavior of the solutions $i_{ \pm}(2.18)$ and (2.19) is modified as

$$
\begin{array}{ll}
i_{+} \sim\left(z-z_{i}\right)^{\eta_{i}(\xi)}, \quad i_{-} \sim\left(z-z_{i}\right)^{1-\eta_{i}(\xi)} /\left(1-2 \eta_{i}(\xi)\right), \\
\bar{i}_{+} \sim\left(\bar{z}-\bar{z}_{i}\right)^{\eta_{i}(\xi)}, \quad & \bar{i}_{-} \sim\left(\bar{z}-\bar{z}_{i}\right)^{1-\eta_{i}(\xi)} /\left(1-2 \eta_{i}(\xi)\right),
\end{array}
$$

where

$$
\eta_{i}(\xi) \equiv \frac{1}{2}-\sqrt{\frac{1}{4}-\frac{\eta_{i}\left(1-\eta_{i}\right)}{\xi^{2}}} .
$$

Thus, all the formulae derived in this subsection, in particular the formulae for the products of Wronskians (3.7)-(3.12), are valid under the following replacement:

$$
p_{i} \rightarrow p_{i}(\xi) \equiv 2 \pi \eta_{i}(\xi) .
$$

Below, we often omit writing the dependence on $\xi$ and denote $p_{i}(\xi)$ simply by $p_{i}$.

\subsection{Poles of Wronskians}

Having introduced the one-parameter deformation, we will next discuss the analytic properties, namely poles and zeros, of the Wronskians with respect to $\xi$. As (3.7)-(3.12) shows, the products of the Wronskians have poles at $\sin p_{i}=0$ and zeros at $\sin \left(\sum_{i} \epsilon_{i} p_{i} / 2\right)=0$, where $\epsilon_{i}$ takes +1 or -1 . Thus, our practical task is to allocate these poles and zeros between two Wronskians.

Let us first discuss poles of the Wronskians. For simplicity, we will only consider poles at $\sin p_{1}=0$. Generalization to other poles is straightforward. At $\sin p_{1}=0$, two eigenvalues of $\Omega_{1}, e^{+i p_{1}}$ and $e^{-i p_{1}}$ become both +1 or both -1 . This, however, does not mean that $\Omega_{1}$ is proportional to the unit matrix at such points: if $\Omega_{1}$ is proportional to the unit matrix, $\Omega_{2}$ must satisfy $\Omega_{2}= \pm \Omega_{3}^{-1}$ owing to the monodromy relation $\Omega_{1} \Omega_{2} \Omega_{3}=1$. However, for generic $p_{2}$ and $p_{3}$, there is no reason for this to be satisfied since $p_{1}, p_{2}$ and $p_{3}$ can be chosen completely independently. Therefore, the only remaining possibility is that $\Omega_{1}$ becomes a Jordan-block:

$$
\Omega_{1} \sim \pm\left(\begin{array}{ll}
1 & c \\
0 & 1
\end{array}\right) \quad \text { at } \sin p_{1}=0 .
$$

This means two eigenvectors of $\Omega_{1}$ degenerate at $\sin p_{1}=0$.

\footnotetext{
${ }^{12}$ Later, utilizing this analogy, we will discuss the WKB expansion of (3.13).
} 
To see what happens at $\sin p_{1}=0$ more explicitly, let us study the behavior of $1_{ \pm}$ near $z_{1}$. Owing to the normalization conditions (3.14), the expansion of $1_{ \pm}$around $z_{1}$ is in general given by

$$
\begin{aligned}
& 1_{+}=\left(z-z_{1}\right)^{\eta_{1}(\xi)}\left(1+a_{+}^{(1)}\left(z-z_{1}\right)+a_{+}^{(2)}\left(z-z_{1}\right)^{2}+\cdots\right) \\
& 1_{-}=\frac{\left(z-z_{1}\right)^{1-\eta_{1}(\xi)}}{1-2 \eta_{1}(\xi)}\left(1+a_{-}^{(1)}\left(z-z_{1}\right)+a_{-}^{(2)}\left(z-z_{1}\right)^{2}+\cdots\right),
\end{aligned}
$$

where $a_{ \pm}^{(n)}$ are functions of $\xi$. An important observation here is that, although $1_{+}$and $1_{-}$ degenerate at $\sin p_{1}=0$, their leading terms, $\left(z-z_{1}\right)^{\eta_{1}}$ and $\left(z-z_{1}\right)^{1-\eta_{1}} /\left(1-2 \eta_{1}\right)$, do not degenerate in general. This apparent contradiction can be resolved if and only if one of $1_{ \pm}$appears in the expansion of the other with a divergent coefficient. More precisely, we expect one of the following two situations is realized at $\sin p_{1}=0$ :

$$
\begin{aligned}
1_{+} & =\left(z-z_{1}\right)^{\eta_{1}(\xi)}+\cdots+A(\xi) 1_{-}+\cdots, \\
A(\xi) & \rightarrow \infty \quad \text { at } \sin p_{1}=0,
\end{aligned}
$$

or

$$
\begin{aligned}
1_{-} & =\frac{\left(z-z_{1}\right)^{1-\eta_{1}(\xi)}}{1-2 \eta_{1}(\xi)}+\cdots+B(\xi) 1_{+}+\cdots, \\
B(\xi) & \rightarrow \infty \quad \text { at } \sin p_{1}=0 .
\end{aligned}
$$

The first case (3.21) is realized if $1_{+}$is bigger than $1_{-}$in the neighborhood of $z_{1}$, namely if $\eta_{1}(\xi)$ is smaller than $1-\eta_{1}(\xi)$. On the other hand, the second case (3.22) is realized if $1_{+}$is smaller than $1_{-}$around $z_{1}$ and $\eta_{1}(\xi)$ is bigger than $1-\eta_{1}(\xi)$. From this simple analysis, we can conclude that, among two solutions $i_{ \pm}$, the solution which is bigger around $z_{i}$ diverges at $\sin p_{i}=0$ whereas the solution which is smaller is finite. This leads to the following rules for determination of poles of the Wronskians:

$$
\text { At } \sin p_{i}=0 \quad\left[\begin{array}{ll}
\text { if } \eta_{i}(\xi)<1 / 2 \Rightarrow\left\langle i_{+}, \bullet\right\rangle=\infty, & \left\langle i_{-}, \bullet\right\rangle \neq \infty, \\
\text { if } \eta_{i}(\xi) \geq 1 / 2 \Rightarrow\left\langle i_{+}, \bullet\right\rangle \neq \infty, & \left\langle i_{-}, \bullet\right\rangle=\infty .
\end{array}\right.
$$

Note that, at the boundary value $\eta_{i}(\xi)=1 / 2$, the Wronskians containing $i_{-}$diverge owing to the explicit factor $1 /\left(1-2 \eta_{i}(\xi)\right)$ contained in (3.14).

\subsection{Zeros of Wronskians}

Having determined the pole structures, let us next discuss zeros of the Wronskians. The determination of zeros is substantially more difficult than the determination of poles since zeros are determined by the global properties on the Riemann surface while the poles are determined solely by the local properties around the punctures. As shown in the previous works $[28,29,31,34,37$, the WKB curve is one of the central tools to explore such global properties. However, as its name suggests, the WKB curve is useful only when we can approximate the solutions to the auxiliary linear problem by the leading term in the WKB expansion. For this reason, it is not powerful enough to fully determine the zeros of the 
Wronskians in the whole spectral parameter region. In this subsection, we shall introduce an appropriate generalization of the WKB curve, to be called the exact WKB curve, which allows us to determine zeros of the Wronskian on the whole parameter plane.

\subsubsection{WKB approximation and WKB curves}

Before introducing the generalized version, let us briefly review the ordinary WKB curves defined in [34]. When $\xi$ is sufficiently close to zero, we can construct approximate solutions to the auxiliary linear problem by the WKB expansion. For instance, the approximate solutions around the singularity $z_{i}$ can be constructed as follows:

$$
\psi \sim \exp \left( \pm \frac{1}{\xi} \int_{z_{i}+\epsilon_{i}}^{z} \sqrt{-T} d z\right)
$$

where $\epsilon_{i}(\ll 1)$ is the regularization parameter. (3.24) gives two different approximate solutions depending on the choice of the sign. One of the solutions is the small solution, which decreases exponentially as it approaches $z_{i}$. The other is the big solution, which increases exponentially as it approaches $z_{i}$. Now let us define the WKB curves as the curves along which the phase of the leading term (3.24) in the WKB expansion is constant. More explicitly, they are given by

$$
\operatorname{Im}\left(\frac{\sqrt{-T}}{\xi} d z\right)=0 .
$$

By analyzing (3.25), we can confirm that there are three different structures of the WKB curves. First, at generic points on the worldsheet, the WKB curves are completely smooth and non-intersecting. Second, at a puncture, the WKB curves radiate in all direction from the puncture. Third, at a zero of $T(z)$, there are three special WKB curves ${ }^{13}$ which radiate from the zero and separate three different configurations of the WKB curves. For a detail, see figure 1. Along the WKB curve, the magnitude of the leading term in the WKB expansion (3.24) monotonically increases or monotonically decreases until they reach a zero or a pole of $T(z)$. Thus, if two punctures $z_{i}$ and $z_{j}$ are connected by a WKB curve and the spectral parameter $\xi$ is sufficiently small, the small solution defined around $z_{i}$, to be denoted $s_{i}$, will grow exponentially as it approaches the other puncture $z_{j}$. This means that the small solution $s_{i}$ behaves like the big solution around $z_{j}$. Therefore it will be linearly independent of $s_{j}$, the small solution defined at $z_{j}$. Consequently, the Wronskian between these two small solutions $\left\langle s_{i}, s_{j}\right\rangle$ will be nonzero. Applying this logic, we conclude that the Wronskians $\left\langle i_{ \pm}, j_{ \pm}\right\rangle$are nonzero if the following three conditions are satisfied: first, two punctures $z_{i}$ and $z_{j}$ are connected by the WKB curves. Second, two eigenvectors $i_{ \pm}$and $j_{ \pm}$ are both small solutions. Third, the approximation by the leading WKB solutions (3.24) is sufficiently good.

\subsubsection{Exact WKB curves}

Evidently, the above analysis is valid only in a restricted region on the spectral parameter plane where the approximation by the leading term in the WKB expansion is reliable.

\footnotetext{
${ }^{13}$ These special WKB curves are called Stokes lines in the literature.
} 


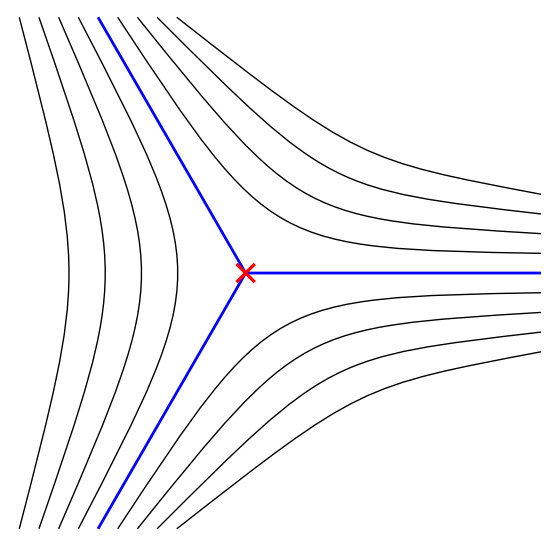

Zero

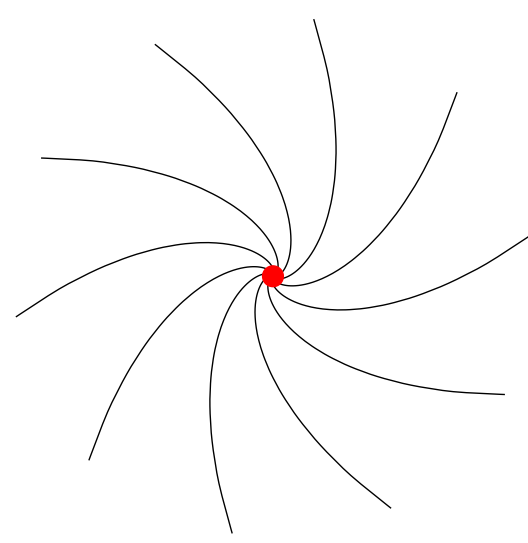

Pole

Figure 1. Structures of the WKB curves around zeros and poles. There are three WKB curves radiating from a zero, while infinitely many WKB curves emanate from a pole. The exact WKB curves, to be discussed later, also have similar structures.

To understand the structure of zeros on the whole spectral parameter plane, we need to generalize the notion of WKB curves.

To motivate our definition of the exact WKB curves, let us first make a small detour and discuss the general structure of the WKB expansions. If we express the solution to the auxiliary linear problem (3.13) as

$$
\psi(z, \xi)=\exp \left(\int_{z_{0}}^{z} P\left(z^{\prime}, \xi\right) d z^{\prime}\right), \quad P(z, \xi)=\sum_{n=-1}^{\infty} \xi^{n} P_{n}(z)
$$

we obtain the following Riccati equation for $P(z ; \xi)$ :

$$
P^{2}+\partial P+\frac{1}{\xi^{2}} T(z)=0 .
$$

Since the left hand side of this equation must vanish at each order of $\xi$ expansion, $P_{n}$ can be determined recursively,

$$
P_{-1}^{2}=-T, \quad P_{n}=\frac{-1}{2 P_{-1}}\left(\sum_{m=0}^{n-1} P_{m} P_{n-m-1}+\partial P_{n-1}\right), \quad n \geq 0 .
$$

Then, dividing $P$ into the odd powers and the even powers of $\xi$,

$$
P=P_{\text {odd }}+P_{\text {even }}, \quad P_{\text {odd }}=\sum_{n=0}^{\infty} \xi^{2 n-1} P_{2 n-1}, \quad P_{\text {even }}=\sum_{n=0}^{\infty} \xi^{2 n} P_{2 n},
$$

we obtain the following relation,

$$
P_{\text {even }}=-\frac{1}{2} \partial \log P_{\text {odd }} .
$$


Using (3.30), we can express the solutions only in terms of $P_{\text {odd }}$. In summary, the WKB expansion of two independent solutions to the auxiliary linear problem can be expressed in the following simple form:

$$
\psi_{\mathrm{WKB}}^{( \pm)}=\frac{1}{\sqrt{P_{\mathrm{WKB}}(z, \xi)}} \exp \left( \pm \int_{z_{0}}^{z} P_{\mathrm{WKB}}\left(z^{\prime}, \xi\right) d z^{\prime}\right),
$$

where $P_{\mathrm{WKB}}$ in (3.31) denotes $P_{\text {odd }}$ in the preceding equations.

Motivated by the simple form of (3.31), let us express the exact solutions to the auxiliary linear problem as

$$
\psi=\frac{1}{\sqrt{2 P_{\mathrm{ex}}(z ; \xi)}} \exp \left(-\int_{z_{0}}^{z} P_{\mathrm{ex}}(z ; \xi) d z\right) .
$$

Although (3.31) and (3.32) are almost identical in form, there is a notable difference: while $P_{\text {WKB }}$ is given only by an asymptotic series with respect to $\xi$ and is ambiguous in a non-perturbative sense, $P_{\text {ex }}$ is defined unambiguously as it is directly defined by the exact solution, $\psi$. Of course, if we expand $P_{\text {ex }}$ perturbatively with respect $\xi$, we will get the same expansion as $P_{\mathrm{WKB}}$. Therefore, $P_{\mathrm{ex}}$ can be regarded as a non-perturbative completion of $P_{\mathrm{WKB}}$. One of the virtues of the expression (3.32) is that we can easily construct another solution satisfying $\langle\psi, \tilde{\psi}\rangle=1$ as

$$
\tilde{\psi}=\frac{1}{\sqrt{2 P_{\mathrm{ex}}(z ; \xi)}} \exp \left(+\int_{z_{0}}^{z} P_{\mathrm{ex}}(z ; \xi) d z\right) .
$$

Using (3.32), let us now discuss the generalization of the WKB curves. The quantity we used to define the original WKB curves, $\sqrt{-T} / \xi$, is the leading term in the expansion of $P_{\text {WKB }}$. Therefore, the most natural generalization of the WKB curves would be to use $P_{\text {ex }}$, which is a non-perturbative completion of $P_{\mathrm{WKB}}$ and define the curves by

$$
\operatorname{Im}\left(P_{\mathrm{ex}}(z ; \xi) d z\right)=0 .
$$

However, this definition is ambiguous since a different choice of the exact solution $\psi$ leads to a different $P_{\mathrm{ex}}$ and thus to different curves. To fix such ambiguities, we define the generalization of the WKB curves, to be called exact WKB curves, separately for each puncture:

Exact WKB curves. Exact WKB curves for the puncture $z_{i}$, to be denoted $\mathrm{eWKB}_{(i)}$, are defined by

$$
\operatorname{Im}\left(P_{\mathrm{ex}}^{(i)}(z ; \xi) d z\right)=0
$$

where $P_{\mathrm{ex}}^{(i)}$ is the exponential factor for the smaller eigenvector among $i_{ \pm}$. More explicitly, it is defined by

$$
s_{i}=\frac{1}{\sqrt{2 P_{\mathrm{ex}}^{(i)}(z ; \xi)}} \exp \left(-\int_{z_{i}+\epsilon_{i}}^{z} P_{\mathrm{ex}}^{(i)}(z ; \xi) d z\right),
$$

where $s_{i}$ denotes the smaller solution among $i_{ \pm}$and $\epsilon_{i}(\ll 1)$ is the parameter we introduced for the regularization. 
Let us now make several comments. First, it is easy to see that this definition of the exact WKB curves reduces to that of the ordinary WKB curves when $\xi$ is close to zero. Second, if we flip the sign in the exponent of (3.36) as

$$
b_{i} \equiv \frac{1}{\sqrt{2 P_{\mathrm{ex}}^{(i)}(z ; \xi)}} \exp \left(+\int_{z_{i}+\epsilon_{i}}^{z} P_{\mathrm{ex}}^{(i)}(z ; \xi) d z\right),
$$

we obtain another solution $b_{i}$, which is big near the puncture $z_{i}$ and satisfies $\left\langle s_{i}, b_{i}\right\rangle=1$. The solution $b_{i}$, constructed in this way, is not guaranteed to be an eigenvector of $\Omega_{i}$. The other eigenvector is in general given by some linear combination of $s_{i}$ and $b_{i}, b_{i}+c s_{i}$. However, as $s_{i}$ is exponentially smaller than $b_{i}$ near $z_{i}, b_{i}$ coincide, up to exponentially small corrections, with the eigenvector in the neighborhood of $z_{i}$.

When $\xi$ is not close to zero, $P_{\mathrm{ex}}^{(i)}$ and $P_{\mathrm{ex}}^{(j)}$ will in general differ by the terms nonperturbative with respect to $\xi$. Therefore $\operatorname{eWKB}_{(i)}$ and $\operatorname{eWKB}_{(j)}$ will be different. Nevertheless, in a sufficient neighborhood of $z_{i}$ or $z_{j}$, we expect $\operatorname{eWKB}_{(i)}$ and $\operatorname{eWKB}_{(j)}$ to be identical. To see this, let us consider the behavior of the small solution $s_{i}$ along $\operatorname{eWKB}_{(i)}$. Along $\mathrm{eWKB}_{(i)}$, the phase of the exponential factor in $s_{i}$ is constant and its magnitude monotonically increases ${ }^{14}$ until it reaches some endpoint. Then, if the punctures $z_{i}$ and $z_{j}$ are connected by some curves in $\operatorname{eWKB}_{(i)}, s_{i}$ will grow exponentially as it approaches $z_{j}$ along the curves, which means that $s_{i}$ behaves like the big solution around $z_{j}$. Since requiring a solution to be exponentially big around a puncture does not uniquely specify the solution, $s_{i}$ is in general expressed as $s_{i} \propto b_{j}+c s_{j}$, where $c$ is some unknown constant. However, since $s_{j}$ is exponentially smaller than $b_{j}$ around $z_{j}$, we can neglect the second term $c s_{j}$ and approximate $s_{i}$ in the neighborhood of $z_{j}$ as

$$
s_{i} \propto b_{j}=\frac{1}{\sqrt{2 P_{\mathrm{ex}}^{(j)}(z ; \xi)}} \exp \left(+\int_{z_{j}+\epsilon_{j}}^{z} P_{\mathrm{ex}}^{(j)}(z ; \xi) d z\right),
$$

up to exponentially small corrections. From (3.38), we can show that $P_{\mathrm{ex}}^{(i)} \sim-P_{\mathrm{ex}}^{(j)}$ near the puncture $z_{j}$. Then, since the exact WKB curves do not depend on the overall sign of $P_{\text {ex }}^{(i)}$, we conclude $\operatorname{eWKB}_{(i)}$ and $\operatorname{eWKB}_{(j)}$ coincide in the vicinity of $z_{j}$.

Let us now use the exact WKB curves to determine the analyticities of the Wronskians. Following exactly the same logic as in the case of the ordinary WKB curves, we can conclude that the Wronskian involving two small solutions $s_{i}$ and $s_{j}$ must be nonzero if two punctures $z_{i}$ and $z_{j}$ are connected by some exact WKB curves. However, unlike the ordinary WKB curves, it is difficult to determine the configuration of the exact WKB curves since the explicit form of $P_{\mathrm{ex}}^{(i)}$ cannot be obtained unless we solve the auxiliary linear problem explicitly. Nevertheless, we shall show below that the local properties of the exact WKB curves, in particular the "number densities" of the curves emanating from

\footnotetext{
${ }^{14}$ Strictly speaking, the small eigenvector (3.36) also contains a prefactor in front of the exponential. This prefactor, however, does not play a significant role in our discussion since it drops out if we consider the ratio of two solutions $s_{i} / b_{i}$. It is in fact sufficient to know the ratio in order to identify the small solution and the big solution.
} 
punctures, turn out to give enough restrictions to determine the full topology (connectivity) of the curves. To see this, let us first give a definition of the number density of the exact WKB curves. Since the exact WKB curves are defined as curves on which $\operatorname{Im} \int P_{\mathrm{ex}}^{(i)} d z$ is constant, the number density of the exact WKB curves emanating from $z_{i}$ can be counted by measuring how $\operatorname{Im} \int P_{\mathrm{ex}}^{(i)} d z$ changes as we go around the puncture. This leads to the following formula:

$$
N_{i} \equiv \frac{1}{2 \pi} \oint_{\mathcal{C}_{i}}\left|\operatorname{Im} P_{\mathrm{ex}}^{(i)} d z\right|,
$$

where the integration contour $\mathcal{C}_{i}$ is an infinitesimal circle encircling the puncture $z_{i}$ counterclockwise. From the asymptotic behavior of $i_{ \pm}$(3.14), we can determine the behavior of $P_{\mathrm{ex}}^{(i)}$ as follows:

$$
P_{\mathrm{ex}}^{(i)} \sim \pm\left(\eta_{i}(\xi)-\frac{1}{2}\right) \frac{1}{z-z_{i}} \quad \text { as } z \rightarrow z_{i}
$$

where + or - sign is chosen depending on which of $i_{ \pm}$is small. Then $N_{i}$ can be computed explicitly as

$$
N_{i} \equiv\left|\operatorname{Re}\left(\eta_{i}(\xi)-\frac{1}{2}\right)\right|
$$

Next, we shall derive two important properties of the $\mathrm{eWKB}_{(i)}$ 's which will be necessary to determine their configurations. Let us first show the following property:

Non-contractibility. All the exact WKB curves which start and end at the same puncture are non-contractible. In other words, such curves go around a different puncture at least once.

To understand this, recall that the Wronskians between small solutions should be nonzero if two punctures are connected by the exact WKB curves. Then, if there exists a contractible cycle connecting the same puncture $z_{i}$, the Wronskian between two identical small solutions $\left\langle s_{i}, s_{i}\right\rangle$ must be nonzero. However, this obviously contradicts the definition of the Wronskian. On the other hand, if the curve connecting the same puncture is non-contractible, namely if the curve goes around a different puncture at least once, we need to take into account the effect of the monodromy $\tilde{\Omega}$ around the punctures and the Wronskian which should be nonzero is now replaced by $\left\langle s_{i}, \tilde{\Omega} s_{i}\right\rangle$. This does not lead to any contradiction and thus we conclude the curves which connect the same puncture should always be noncontractible. The next property to show is given as follows:

Endpoints. All but finitely many exact WKB curves terminate at punctures.

To derive this, let us first classify possible endpoints of the exact WKB curves. As in the case of ordinary WKB curves, possible endpoints are zeros or poles of $P_{\mathrm{ex}}^{(i)}$. Among these two, zeros are unimportant since the number of the exact WKB curves flowing into zeros is always finite as shown in figure 1. On the other hand, a pole can be an endpoint for infinitely many curves and thus plays a crucial role in the analysis. There are basically three 
different types for poles. The first type is the punctures, at which the vertex operators are inserted. The second type is the singularities of the small eigenvector $s_{i}$, different from the punctures. However, since the auxiliary linear equation we are solving is of the Fuchsian type, we expect $s_{i}$ becomes singular only at the punctures and other singularities are absent. The last type is the zeros of the eigenvector $s_{i}$. Although $s_{i}$ in general has several zeros on the Riemann surface, such points cannot be the endpoints of the exact WKB curves for the following reason: at zeros of $s_{i}$, the ratio between $s_{i}$ and $b_{i}, s_{i} / b_{i}$, must also be zero. ${ }^{15}$ However, this contradicts the basic property of the exact WKB curve that such a ratio, determined by the exponential factor in (3.36), monotonically increases along the exact WKB curve as we move away from $z_{i}$. From these considerations, we can conclude that all but finitely many exact WKB curves end at punctures.

Having proven two important properties, now we are in a position to discuss the configuration of the exact WKB curves. There are essentially two different cases. First consider the case where $N_{i}$ 's satisfy the triangle inequality:

$$
N_{i}+N_{j}-N_{k}>0
$$

In this case, to be called the symmetric case, the only allowed configuration which obeys the properties derived above is the one in which three punctures are piece-wise connected (see figure 2). Next consider the case where the following triangular inequality is violated:

$$
N_{2}+N_{3}-N_{1}<0
$$

Note that all the remaining cases can be obtained from (3.43) by the permutation of three indices 1,2 and 3 . These cases are called the asymmetric cases. In the case (3.43), the only consistent way to connect three punctures is to arrange the curves so that all the curves emanating from $z_{2}$ and $z_{3}$ end at $z_{1}$ and some of the curves emanating from $z_{1}$ go back to $z_{1}$ by going around $z_{2}$ or $z_{3}$ (see figure 2 ).

In this way, we can completely determine the configuration of the exact WKB curves by the local data of the auxiliary linear problem. Below, we will see explicitly how the configuration of the exact WKB curves can be used to determine zeros of the Wronskians.

\subsubsection{Determination of zeros}

To illustrate the outline of the discussion with an explicit example, let us consider the factor,

$$
\sin \left(\frac{p_{1}+p_{2}+p_{3}}{2}\right)
$$

and determine which of the Wronskian become zero when (3.44) vanishes. From (3.7)(3.12), the products of the Wronskians which vanish are given by

$$
\left\langle 1_{+}, 2_{+}\right\rangle\left\langle 1_{-}, 2_{-}\right\rangle, \quad\left\langle 2_{+}, 3_{+}\right\rangle\left\langle 2_{-}, 3_{-}\right\rangle, \quad\left\langle 3_{+}, 1_{+}\right\rangle\left\langle 3_{-}, 1_{-}\right\rangle .
$$

\footnotetext{
${ }^{15} b_{i}$ must be nonzero at such points to ensure the normalization condition $\left\langle s_{i}, b_{i}\right\rangle=1$.
} 


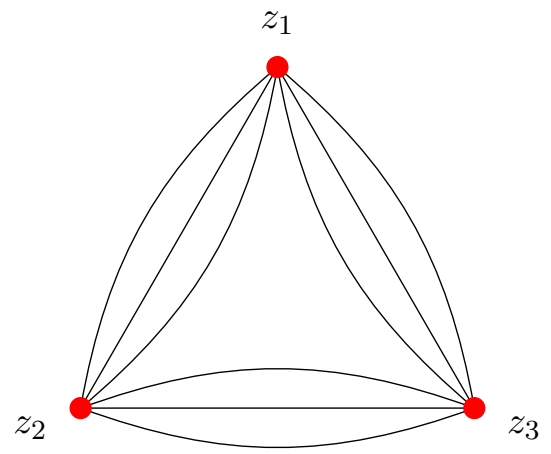

(a) Symmetric case $\left(N_{i}+N_{j}-N_{k}>0\right)$

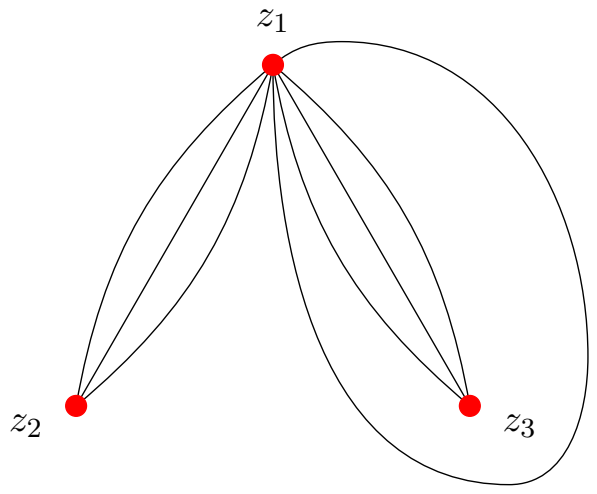

(b) Asymmetric case $\left(N_{2}+N_{3}-N_{1}<0\right)$

Figure 2. Two types of configurations of the exact WKB curves. In the symmetric case, all the punctures are connected to each other by exact WKB curves. In the asymmetric case, we have non-contractible exact WKB curves which start and end at the same puncture. As the exact WKB curves are non-intersecting, there exists a pair of punctures between which there are no connecting curves in such a case.

An important feature of (3.45) is that, if we group the eigenvectors into $\left\{1_{+}, 2_{+}, 3_{+}\right\}$and $\left\{1_{-}, 2_{-}, 3_{-}\right\}$, only the Wronskians within the same group appear in (3.45). This is in fact quite a general feature and is true also for other situations.

Now, let us prove two theorems, which will be useful for determination of zeros. The first theorem is the following one, which we have already proven:

Theorem 1. When two punctures $z_{i}$ and $z_{j}$ are connected by the exact WKB curve, the Wronskian between two small eigenvectors $\left\langle s_{i}, s_{j}\right\rangle$ is nonzero.

Before writing down the second theorem, let us prove the following useful lemma:

Lemma. In (3.45), only one of the two Wronskians in each product vanishes. The other does not vanish.

To prove Lemma, first note that all the zeros of (3.44) are simple zeros. Note also that the eigenvectors and their Wronskians are in general single-valued around the zeros of (3.44). From these two facts, it follows that only one of the two Wronskians in the product vanishes at the zeros of (3.44). If both of the Wronskians vanish, (3.44) must have a double zero and contradicts the aforementioned property. Now, using these lemmas, we can derive the following important theorem.

Theorem 2. There are only two distinct possibilities concerning zeros of the Wronskians in (3.45):

1. All the Wronskians among $\left\{1_{+}, 2_{+}, 3_{+}\right\}$are zero and all the Wronskians among $\left\{1_{-}, 2_{-}, 3_{-}\right\}$are nonzero.

2. All the Wronskians among $\left\{1_{+}, 2_{+}, 3_{+}\right\}$are nonzero and all the Wronskians among $\left\{1_{-}, 2_{-}, 3_{-}\right\}$are zero. 
This theorem can be proven in the following way. First, assume that two of the three Wronskians in $\left\{1_{+}, 2_{+}, 3_{+}\right\}$are zero. In such a case, all $i_{+}$'s become proportional to each other. Then, it follows that the remaining one Wronskian also vanishes. Using this line of reasoning and the results of Lemma, we can easily show that there are only two distinct possibilities, described in Theorem 2.

Using the theorems we just proved, we can determine zeros of the Wronskians at relative ease when the configuration of the exact WKB curve is symmetric. In such cases, one of the groups, $\left\{1_{+}, 2_{+}, 3_{+}\right\}$and $\left\{1_{-}, 2_{-}, 3_{-}\right\}$, contains at least two small eigenvectors. Then, applying Theorem 1 and Theorem 2, we can immediately conclude that the Wronskians from such a group are all nonzero whereas the Wronskians from the other group are all zero. Let us next consider the asymmetric case. For simplicity, let us assume that $N_{1}>N_{2}+N_{3}$ is satisfied. ${ }^{16}$ In such a case, there exist exact WKB curves which start $z_{1}$, go around $z_{2}$ (or $z_{3}$ ) and return to $z_{1}$. Therefore, to utilize the information on the exact WKB curves, it is important to consider the Wronskians,

$$
\left\langle 1_{+}, \Omega_{2} 1_{+}\right\rangle, \quad\left\langle 1_{-}, \Omega_{2} 1_{-}\right\rangle .
$$

To compute these Wronskians, we first note $1_{ \pm}$can be expressed in terms of $2_{ \pm}$as follows:

$$
1_{ \pm}=\left\langle 1_{ \pm}, 2_{-}\right\rangle 2_{+}-\left\langle 1_{ \pm}, 2_{+}\right\rangle 2_{-} .
$$

Then, applying $\Omega_{2}$ to (3.47) and substituting them to (3.46), we can express (3.46) in terms of the ordinary Wronskians as

$$
\begin{aligned}
& \left\langle 1_{+}, \Omega_{2} 1_{+}\right\rangle=2 i \sin p_{2}\left\langle 1_{+}, 2_{-}\right\rangle\left\langle 1_{+}, 2_{+}\right\rangle, \\
& \left\langle 1_{-}, \Omega_{2} 1_{-}\right\rangle=2 i \sin p_{2}\left\langle 1_{-}, 2_{-}\right\rangle\left\langle 1_{-}, 2_{+}\right\rangle .
\end{aligned}
$$

Since $\Omega_{2} 1_{+}$can be obtained by parallel-transporting $1_{+}$along the exact WKB curve which starts and ends at $z_{1}, \Omega_{2} 1_{+}$behaves as the big solution around $z_{1}$ when $1_{+}$is the small solution. Therefore, the Wronskian $\left\langle 1_{+}, \Omega_{2} 1_{+}\right\rangle$is nonzero in this case. Then, because of the expression (3.48), $\left\langle 1_{+}, 2_{+}\right\rangle$is also nonzero. Consequently, using Theorem 2, we can show that the Wronskians among $\left\{1_{+}, 2_{+}, 3_{+}\right\}$are nonzero and the Wronskians among $\left\{1_{-}, 2_{-}, 3_{-}\right\}$are zero. Similarly, we can show that the Wronskians among $\left\{1_{-}, 2_{-}, 3_{-}\right\}$are nonzero and the Wronskians among $\left\{1_{+}, 2_{+}, 3_{+}\right\}$are zero when $1_{-}$is the small eigenvector.

Performing a similar analysis also for other zeros, we can derive the following general rules:

\section{Decomposition of the eigenvectors into two groups.}

When a factor of the form $\sin \left(\sum_{i} \epsilon_{i} p_{i} / 2\right)$ vanishes, the Wronskians which vanish are the ones among $\left\{1_{\epsilon_{1}}, 2_{\epsilon_{2}}, 3_{\epsilon_{3}}\right\}$ and the ones among $\left\{1_{-\epsilon_{1}}, 2_{-\epsilon_{2}}, 3_{-\epsilon_{3}}\right\}$.

\section{Symmetric case.}

When the configuration of the exact WKB curves is symmetric, the Wronskians from the group which contains two or more small solutions are nonzero whereas the Wronskians from the other group are zero.

\footnotetext{
${ }^{16}$ Generalization to other cases is straightforward.
} 


\section{Asymmetric case.}

When the configuration of the exact WKB curves is asymmetric and $N_{i}$ 's satisfy $N_{i}>N_{j}+N_{k}$, the Wronskians from the group which contains the smaller solution of $i_{ \pm}$are nonzero whereas the Wronskians from the other group are zero.

Let us now examine the above rules more in detail. For simplicity, let us focus on $\left\langle 1_{+}, 2_{+}\right\rangle$and consider the zeros which arise when $\sin \left(\sum_{i} p_{i} / 2\right)$ vanishes. To apply the general rules, we need to know 1) Which of $i_{ \pm}$is small and 2) The configuration of the exact WKB curves. What is important in the following discussion is that both of these are determined by the real part of $\eta_{i}(\xi)-1 / 2$. In fact, the relative magnitude of $i_{ \pm}$is determined as

$$
\begin{aligned}
& \operatorname{Re}\left(\eta_{i}(\xi)-\frac{1}{2}\right)>0 \quad \Rightarrow \quad i_{+}: \text {small, } i_{-}: \text {big }, \\
& \operatorname{Re}\left(\eta_{i}(\xi)-\frac{1}{2}\right)<0 \quad \Rightarrow \quad i_{+}: \text {big }, i_{-}: \text {small },
\end{aligned}
$$

and the numbers of the exact WKB curves $N_{i}$ are given in terms of $\eta_{i}(\xi)-1 / 2$ as shown in (3.41). Below we will denote this important quantity by $\gamma_{i}, \gamma_{i} \equiv \operatorname{Re}\left(\eta_{i}(\xi)-1 / 2\right)$. Let us first consider the case where all $\gamma_{i}$ 's are positive. In this case, $1_{+}, 2_{+}$and $3_{+}$become all small and $\left\langle 1_{+}, 2_{+}\right\rangle$is nonzero irrespective of the configuration of the exact WKB curves. Next consider the case where only $\gamma_{3}$ is negative and $3_{+}$is a big solution. In this case, $\left\langle 1_{+}, 2_{+}\right\rangle$vanishes if and only if $N_{3}>N_{1}+N_{2}$ is satisfied. This condition translates into the following condition for $\gamma_{i}: \gamma_{1}+\gamma_{2}+\gamma_{3}<0$. Similarly, in the case where only $\gamma_{1}$ (or $\gamma_{2}$ ) is negative, $\left\langle 1_{+}, 2_{+}\right\rangle$vanishes when $N_{1}>N_{2}+N_{3}$ (or $N_{2}>N_{3}+N_{1}$ ) is satisfied. This condition also translates into the same condition, $\gamma_{1}+\gamma_{2}+\gamma_{3}<0$. One can perform this kind of analysis also for the cases where two or more $\gamma_{i}$ 's are negative and, as a result, we find that zeros of $\left\langle 1_{+}, 2_{+}\right\rangle$associated with $\sin \left(\sum_{i} p_{i} / 2\right)=0$ are completely determined by the sign of $\gamma_{1}+\gamma_{2}+\gamma_{3}$. Re-expressing this condition in terms of $p_{i}$ 's, we obtain the following rule

$$
\begin{aligned}
& \operatorname{Re}\left(\frac{p_{1}+p_{2}+p_{3}}{2}\right)>\frac{3 \pi}{2} \quad \Rightarrow \quad\left\langle 1_{+}, 2_{+}\right\rangle \neq 0, \\
& \operatorname{Re}\left(\frac{p_{1}+p_{2}+p_{3}}{2}\right)<\frac{3 \pi}{2} \quad \Rightarrow \quad\left\langle 1_{+}, 2_{+}\right\rangle=0 .
\end{aligned}
$$

Applying this logic to other situations, we can get explicit rules to determine zeros of the Wronskians.

Now, let us write down the final result combining the results for poles and the results for zeros. For simplicity, here we just present the result for $\left\langle 1_{ \pm}, 2_{ \pm}\right\rangle$, but the analyticity 
of other Wronskians can be easily obtained by the permutation of indices, 1, 2 and 3 .

Analyticity of $\left\langle\mathbf{1}_{+}, \mathbf{2}_{+}\right\rangle$

Poles: at $\sin p_{1}=0$ if $\operatorname{Re} p_{1}<\pi$, and at $\sin p_{2}=0$ if $\operatorname{Re} p_{2}<\pi$,

Zeros: at $\sin \frac{p_{1}+p_{2}-p_{3}}{2}=0$ if $\operatorname{Re} \frac{p_{1}+p_{2}-p_{3}}{2}<\frac{\pi}{2}$,

$$
\text { and at } \sin \frac{p_{1}+p_{2}+p_{3}}{2}=0 \text { if } \operatorname{Re} \frac{p_{1}+p_{2}+p_{3}}{2}<\frac{3 \pi}{2} \text {. }
$$

Analyticity of $\left\langle 1_{-}, 2_{-}\right\rangle$

Poles: at $\sin p_{1}=0$ if $\operatorname{Re} p_{1} \geq \pi$, and at $\sin p_{2}=0$ if $\operatorname{Re} p_{2} \geq \pi$,

Zeros: at $\sin \frac{p_{1}+p_{2}-p_{3}}{2}=0$ if $\operatorname{Re} \frac{p_{1}+p_{2}-p_{3}}{2}>\frac{\pi}{2}$,

$$
\text { and at } \sin \frac{p_{1}+p_{2}+p_{3}}{2}=0 \text { if } \operatorname{Re} \frac{p_{1}+p_{2}+p_{3}}{2}>\frac{3 \pi}{2} \text {. }
$$

Analyticity of $\left\langle 1_{+}, 2_{-}\right\rangle$

Poles: at $\sin p_{1}=0$ if $\operatorname{Re} p_{1}<\pi$, and at $\sin p_{2}=0$ if $\operatorname{Re} p_{2} \geq \pi$,

Zeros: at $\sin \frac{-p_{1}+p_{2}+p_{3}}{2}=0$ if $\operatorname{Re} \frac{-p_{1}+p_{2}+p_{3}}{2}>\frac{\pi}{2}$,

$$
\text { and at } \sin \frac{p_{1}-p_{2}+p_{3}}{2}=0 \text { if } \operatorname{Re} \frac{p_{1}-p_{2}+p_{3}}{2}<\frac{\pi}{2} \text {. }
$$

Analyticity of $\left\langle\mathbf{1}_{-}, \mathbf{2}_{+}\right\rangle$

Poles: at $\sin p_{1}=0$ if $\operatorname{Re} p_{1} \geq \pi$, and at $\sin p_{2}=0$ if $\operatorname{Re} p_{2}<\pi$,

Zeros: at $\sin \frac{-p_{1}+p_{2}+p_{3}}{2}=0$ if $\operatorname{Re} \frac{-p_{1}+p_{2}+p_{3}}{2}<\frac{\pi}{2}$,

and at $\sin \frac{p_{1}-p_{2}+p_{3}}{2}=0$ if $\operatorname{Re} \frac{p_{1}-p_{2}+p_{3}}{2}>\frac{\pi}{2}$.

In the next subsection, we will utilize this information to explicitly compute the individual Wronskians.

\subsection{Calculation of Wronskians and three-point functions}

Now let us evaluate Wronskians. To evaluate Wronskians, we need to decompose the right hand sides of (3.7)-(3.12) based on the analyticity properties listed above. To carry this out, we will make use of the following useful formula:

$$
\sin z=\frac{\pi}{\Gamma\left(\frac{z}{\pi}\right) \Gamma\left(1-\frac{z}{\pi}\right)} .
$$

Since the gamma function has poles only at $n \in \mathbb{Z}_{\leq 0}$, (3.56) decomposes the zeros of $\sin z$ into two groups, those on the positive real axis and those on the negative real axis (see figure 3). Then, from the analyticity conditions determined in the previous subsection, we 


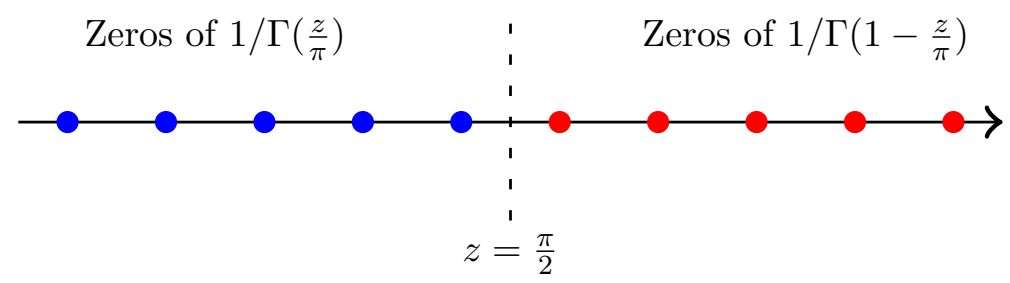

Figure 3. Decomposition of the zeros of $\sin z \cdot 1 / \Gamma\left(1-\frac{z}{\pi}\right)$ contains zeros on the positive real axis whereas $1 / \Gamma\left(\frac{z}{\pi}\right)$ contains zeros on the negative real axis.

obtain the following decompositions:

$$
\begin{aligned}
\left\langle 1_{+}, 2_{+}\right\rangle & \propto \frac{\Gamma\left(p_{1} / \pi\right) \Gamma\left(p_{2} / \pi\right)}{\Gamma\left(\frac{p_{1}+p_{2}-p_{3}}{2 \pi}\right) \Gamma\left(\frac{p_{1}+p_{2}+p_{3}}{2 \pi}-1\right)}, \\
\left\langle 1_{-}, 2_{-}\right\rangle & \propto \frac{\Gamma\left(1-p_{1} / \pi\right) \Gamma\left(1-p_{2} / \pi\right)}{\Gamma\left(1-\frac{p_{1}+p_{2}-p_{3}}{2 \pi}\right) \Gamma\left(2-\frac{p_{1}+p_{2}+p_{3}}{2 \pi}\right)}, \\
\left\langle 1_{+}, 2_{-}\right\rangle & \propto \frac{\Gamma\left(p_{1} / \pi\right) \Gamma\left(1-p_{2} / \pi\right)}{\Gamma\left(1-\frac{-p_{1}+p_{2}+p_{3}}{2 \pi}\right) \Gamma\left(\frac{p_{1}-p_{2}+p_{3}}{2 \pi}\right)}, \\
\left\langle 1_{-}, 2_{+}\right\rangle & \propto \frac{\Gamma\left(1-p_{1} / \pi\right) \Gamma\left(p_{2} / \pi\right)}{\Gamma\left(\frac{-p_{1}+p_{2}+p_{3}}{2 \pi}\right) \Gamma\left(1-\frac{p_{1}-p_{2}+p_{3}}{2 \pi}\right)} .
\end{aligned}
$$

Note that the constants of proportionality in the above equations cannot be determined purely by the analyticity.

To determine the proportionality constants, we study the asymptotic behavior of Wronskians as $\xi$ goes to infinity using the WKB expansion. As the relative magnitude of $i_{ \pm}$ depends on $\operatorname{Arg} \xi$, we choose $\operatorname{Arg} \xi$ appropriately such that $i_{+}$is the small solution and $i_{-}$ is the big solution in the limit $\xi \rightarrow \infty$. In such a case, $i_{+}$can be expressed in terms of $P_{\mathrm{ex}}^{(i)}$ as (3.36). To determine the constant of proportionality in (3.36), we study the behavior around $z_{i}$ and use the approximate form ${ }^{17}$ of $P_{\mathrm{ex}}^{(i)}$ in the vicinity of $z_{i},(3.40)$. Then, the constant of proportionality can be computed by comparing the definition of the right hand side of (3.36) and the normalization of $i_{+}$, given in (3.14). The result is given as follows:

$$
i_{+}=\lim _{\epsilon_{i} \rightarrow 0} \epsilon_{i}^{\eta_{i}(\xi)-1 / 2} \sqrt{\frac{1 / 2-\eta_{i}(\xi)}{P_{\mathrm{ex}}^{(i)}(z, \xi)}} \exp \left(-\int_{z_{i}+\epsilon_{i}}^{z} P_{\mathrm{ex}}^{(i)}\left(z^{\prime}, \xi\right) d z^{\prime}\right) .
$$

Then, approximating $P_{\mathrm{ex}}^{(i)}$ in (3.61) by the leading term of the WKB expansion, we obtain the approximate expression for $i_{+}$at $\xi \rightarrow 0$. However, to write down an explicit expression, we first need to clarify the choice of branch of $\sqrt{-T(z)}$ appearing in the expansion of $P_{\mathrm{ex}}^{(i)}$. In the case of the three-point functions, $\sqrt{-T(z)}$ always has one branch cut and its position depends on the relative magnitudes of $\eta_{i}\left(1-\eta_{i}\right)$. In what follows, we only consider the case where $\eta_{2}\left(1-\eta_{2}\right)$ is larger than $\eta_{1}\left(1-\eta_{1}\right)$ and $\eta_{3}\left(1-\eta_{3}\right)$ and the branch cut is located between $z_{1}$ and $z_{3}$, as shown in figure 4 . In such a case, the branch of $\sqrt{-T(z)}$ on the first

\footnotetext{
${ }^{17}$ In this case, the minus sign must be chosen in (3.40) to reproduce the behavior of $i_{+}$near $z_{i}$.
} 
Figure 4. Branch cut of $\sqrt{-T(z)}$. We choose $\eta_{i}$ such that the branch cut is located between $z_{1}$ and $z_{2}$.

sheet can be chosen as follows:

$$
\begin{aligned}
\sqrt{-T(z)} & \sim \frac{i\left|\sqrt{\eta_{i}\left(1-\eta_{i}\right)}\right|}{z-z_{i}} \quad \text { as } z \rightarrow z_{i} \quad \text { for } i=1,3, \\
& \sim \frac{-i\left|\sqrt{\eta_{i}\left(1-\eta_{i}\right)}\right|}{z-z_{2}} \quad \text { as } z \rightarrow z_{2} .
\end{aligned}
$$

Although the discussion below is restricted to this particular case, the final result will turn out to be completely symmetric under the permutation of $z_{1}, z_{2}$ and $z_{3}$ and is applicable also to other cases.

With this choice of the branch, we can write down approximate expressions for $i_{+}$as

$$
\begin{aligned}
1_{+} & \sim \epsilon_{1}^{\eta_{1}(\xi)-1 / 2} \sqrt{\frac{1}{2}-\eta_{1}(\xi)}\left(\frac{\xi^{2}}{-T}\right)^{1 / 4} \exp \left(-\frac{1}{\xi} \int_{z_{1}+\epsilon_{1}}^{z} \sqrt{-T} d z^{\prime}\right), \\
2_{+} & \sim \epsilon_{2}^{\eta_{2}(\xi)-1 / 2} \sqrt{\frac{1}{2}-\eta_{2}(\xi)}\left(\frac{\xi^{2}}{-T}\right)^{1 / 4} \exp \left(\frac{1}{\xi} \int_{z_{2}+\epsilon_{2}}^{z} \sqrt{-T} d z^{\prime}\right), \\
3_{+} & \sim \epsilon_{3}^{\eta_{3}(\xi)-1 / 2} \sqrt{\frac{1}{2}-\eta_{3}(\xi)}\left(\frac{\xi^{2}}{-T}\right)^{1 / 4} \exp \left(-\frac{1}{\xi} \int_{z_{3}+\epsilon_{3}}^{z} \sqrt{-T} d z^{\prime}\right) .
\end{aligned}
$$

Then the Wronskians which only include $i_{+}$can be calculated as

$$
\begin{aligned}
& \left\langle 1_{+}, 2_{+}\right\rangle \sim \lim _{\epsilon_{i} \rightarrow 0} \epsilon_{1}^{\eta_{1}(\xi)-1 / 2} \epsilon_{2}^{\eta_{2}(\xi)-1 / 2} \sqrt{\left(1-2 \eta_{1}(\xi)\right)\left(1-2 \eta_{2}(\xi)\right)} \exp \left(-\frac{1}{\xi} \int_{z_{1}+\epsilon_{1}}^{z_{2}+\epsilon_{2}} \sqrt{-T} d z^{\prime}\right), \\
& \left\langle 2_{+}, 3_{+}\right\rangle \sim \lim _{\epsilon_{i} \rightarrow 0} \epsilon_{2}^{\eta_{2}(\xi)-1 / 2} \epsilon_{3}^{\eta_{3}(\xi)-1 / 2} \sqrt{\left(1-2 \eta_{2}(\xi)\right)\left(1-2 \eta_{3}(\xi)\right)} \exp \left(-\frac{1}{\xi} \int_{z_{3}+\epsilon_{2}}^{z_{2}+\epsilon_{3}} \sqrt{-T} d z^{\prime}\right), \\
& \left\langle 3_{+}, 1_{+}\right\rangle \sim \lim _{\epsilon_{i} \rightarrow 0} \epsilon_{3}^{\eta_{3}(\xi)-1 / 2} \epsilon_{1}^{\eta_{1}(\xi)-1 / 2} \sqrt{\left(1-2 \eta_{3}(\xi)\right)\left(1-2 \eta_{1}(\xi)\right)} \exp \left(-\frac{1}{\xi} \int_{z_{3}+\epsilon_{3}}^{\hat{z}_{1}+\epsilon_{1}} \sqrt{-T} d z^{\prime}\right),
\end{aligned}
$$


where $\hat{z}_{1}$ in the last line denotes the point on the lower sheet of $y^{2}=-T(z)$ right below $z_{1}$. To evaluate the above formulae, we need to compute the contour integral $\int_{z_{i}}^{z_{j}} \sqrt{-T} d z^{\prime}$. The easiest way to compute them is to map $z_{1}, z_{2}$ and $z_{3}$ to 0,1 and $\infty$ by the $\operatorname{SL}(2, \mathbb{C})$ transformation and then evaluate the integral taking into account the effect of the transformation. The transformation we use is

$$
w \equiv-\frac{z_{23}}{z_{12}} \frac{z-z_{1}}{z-z_{3}},
$$

where $z_{i j} \equiv z_{i}-z_{j}$. The stress-energy tensor in this coordinate is given by

$$
T(w)=\frac{\eta_{1}\left(1-\eta_{1}\right)+\left(\eta_{2}\left(1-\eta_{2}\right)-\eta_{1}\left(1-\eta_{1}\right)-\eta_{3}\left(1-\eta_{3}\right)\right) w+\eta_{3}\left(1-\eta_{3}\right) w^{2}}{w^{2}(w-1)^{2}} .
$$

Then, the Wronskians can be re-expressed as

$$
\begin{aligned}
\left\langle 1_{+}, 2_{+}\right\rangle \sim & \left(\frac{z_{12} z_{31}}{z_{23}}\right)^{\eta_{1}(\xi)-1 / 2}\left(\frac{z_{12} z_{23}}{z_{31}}\right)^{\eta_{2}(\xi)-1 / 2} \sqrt{\left(1-2 \eta_{1}(\xi)\right)\left(1-2 \eta_{2}(\xi)\right)} \\
& \times \lim _{\epsilon_{i} \rightarrow 0} \epsilon_{1}^{\eta_{1}(\xi)-1 / 2} \epsilon_{2}^{\eta_{2}(\xi)-1 / 2} \exp \left(-\frac{1}{\xi} \int_{\epsilon_{1}}^{1+\epsilon_{2}} \sqrt{-T} d w^{\prime}\right), \\
\left\langle 2_{+}, 3_{+}\right\rangle \sim & \left(\frac{z_{12} z_{23}}{z_{31}}\right)^{\eta_{2}(\xi)-1 / 2}\left(\frac{z_{23} z_{31}}{z_{12}}\right)^{\eta_{3}(\xi)-1 / 2} \sqrt{\left(1-2 \eta_{2}(\xi)\right)\left(1-2 \eta_{3}(\xi)\right)} \\
& \lim _{\epsilon_{i} \rightarrow 0} \epsilon_{2}^{\eta_{2}(\xi)-1 / 2} \epsilon_{3}^{\eta_{3}(\xi)-1 / 2} \exp \left(-\frac{1}{\xi} \int_{-\epsilon_{3}^{-1}}^{1+\epsilon_{2}} \sqrt{-T} d w^{\prime}\right), \\
\left\langle 3_{+}, 1_{+}\right\rangle \sim & \left(\frac{z_{23} z_{31}}{z_{12}}\right)^{\eta_{3}(\xi)-1 / 2}\left(\frac{z_{12} z_{31}}{z_{23}}\right)^{\eta_{1}(\xi)-1 / 2} \sqrt{\left(1-2 \eta_{3}(\xi)\right)\left(1-2 \eta_{1}(\xi)\right)} \\
& \lim _{\epsilon_{i} \rightarrow 0} \epsilon_{3}^{\eta_{3}(\xi)-1 / 2} \epsilon_{1}^{\eta_{1}(\xi)-1 / 2} \exp \left(-\frac{1}{\xi} \int_{-\epsilon_{3}^{-1}}^{0} \sqrt{-T} d w^{\prime}\right),
\end{aligned}
$$

where the regularization parameters $\epsilon_{i}$ 's in the above formulae are redefined as

$$
\epsilon_{1}^{\text {new }} \equiv \frac{z_{23}}{z_{12} z_{31}} \epsilon_{1}^{\text {old }}, \quad \epsilon_{2}^{\text {new }} \equiv \frac{z_{31}}{z_{12} z_{23}} \epsilon_{2}^{\text {old }}, \quad \epsilon_{3}^{\text {new }} \equiv \frac{z_{12}}{z_{23} z_{31}} \epsilon_{3}^{\text {old }},
$$

and the prefactors depending on $z_{i j}$ are results of such redefinition. Each contour integral $\int \sqrt{-T} d w$ is divergent in the limit $\epsilon_{i} \rightarrow 0$. However, combined with the prefactors $\epsilon_{*}$, they become completely finite and can be evaluated analytically. Then, by comparing ${ }^{18}$ the resultant asymptotic formula with the $\xi \rightarrow 0$ limit of the left hand side of (3.57) and (3.58), we can finally determine $\left\langle 1_{+}, 2_{+}\right\rangle$and $\left\langle 1_{-}, 2_{-}\right\rangle$as

$$
\begin{aligned}
& \left\langle 1_{+}, 2_{+}\right\rangle=\left(\frac{z_{12} z_{31}}{z_{23}}\right)^{p_{1} / 2 \pi-1 / 2}\left(\frac{z_{12} z_{23}}{z_{31}}\right)^{p_{2} / 2 \pi-1 / 2} \frac{\Gamma\left(p_{1} / \pi\right) \Gamma\left(p_{2} / \pi\right)}{\Gamma\left(\frac{p_{1}+p_{2}-p_{3}}{2 \pi}\right) \Gamma\left(\frac{p_{1}+p_{2}+p_{3}}{2 \pi}-1\right)}, \\
& \left\langle 1_{-}, 2_{-}\right\rangle=\left(\frac{z_{12} z_{31}}{z_{23}}\right)^{1 / 2-p_{1} / 2 \pi}\left(\frac{z_{12} z_{23}}{z_{31}}\right)^{1 / 2-p_{2} / 2 \pi} \frac{\Gamma\left(1-p_{1} / \pi\right) \Gamma\left(1-p_{2} / \pi\right)}{\Gamma\left(1-\frac{p_{1}+p_{2}-p_{3}}{2 \pi}\right) \Gamma\left(2-\frac{p_{1}+p_{2}+p_{3}}{2 \pi}\right)} .
\end{aligned}
$$

\footnotetext{
${ }^{18}$ To compare two expressions, we used the Stirling formula, $\log \Gamma(x) \sim(x-1 / 2) \log x-x$.
} 
Other $\left\langle i_{+}, j_{+}\right\rangle$and $\left\langle i_{-}, j_{-}\right\rangle$are obtained from the permutation of indices. On the other hand, the Wronskians of the type $\left\langle i_{+}, j_{-}\right\rangle$can be obtained by first specifying the explicit form of the monodromy matrices $\Omega_{i}$ using the result for $\left\langle i_{+}, j_{+}\right\rangle$and $\left\langle i_{-}, j_{-}\right\rangle$and then calculating the Wronskians explicitly. The result is

$$
\begin{aligned}
& \left\langle 1_{+}, 2_{-}\right\rangle=\left(\frac{z_{12} z_{31}}{z_{23}}\right)^{p_{1} / 2 \pi-1 / 2}\left(\frac{z_{12} z_{23}}{z_{31}}\right)^{1 / 2-p_{2} / 2 \pi} \frac{\Gamma\left(p_{1} / \pi\right) \Gamma\left(1-p_{2} / \pi\right)}{\Gamma\left(1-\frac{-p_{1}+p_{2}+p_{3}}{2 \pi}\right) \Gamma\left(\frac{p_{1}-p_{2}+p_{3}}{2 \pi}\right)}, \\
& \left\langle 1_{-}, 2_{+}\right\rangle=\left(\frac{z_{12} z_{31}}{z_{23}}\right)^{1 / 2-p_{1} / 2 \pi}\left(\frac{z_{12} z_{23}}{z_{31}}\right)^{p_{2} / 2 \pi-1 / 2} \frac{\Gamma\left(1-p_{1} / \pi\right) \Gamma\left(p_{2} / \pi\right)}{\Gamma\left(\frac{-p_{1}+p_{2}+p_{3}}{2 \pi}\right) \Gamma\left(1-\frac{p_{1}-p_{2}+p_{3}}{2 \pi}\right)} .
\end{aligned}
$$

Using these formulae, we can explicitly calculate $C_{i}$ using (2.33) as

$$
\begin{aligned}
C_{1}=- & \frac{1}{2} \log \lambda-\frac{1-2 \eta_{1}}{2} \log \frac{\left|z_{12}\right|^{2}\left|z_{31}\right|^{2}}{\left|z_{23}\right|^{2}} \\
& -\frac{1}{2} \log \frac{\gamma\left(\eta_{1}+\eta_{2}-\eta_{3}\right) \gamma\left(\eta_{3}+\eta_{1}-\eta_{2}\right) \gamma\left(\eta_{1}+\eta_{2}+\eta_{3}-1\right)}{\gamma\left(2 \eta_{1}\right)^{2} \gamma\left(\eta_{2}+\eta_{3}-\eta_{1}\right)}, \\
C_{2}= & \frac{1}{2} \log \lambda-\frac{1-2 \eta_{2}}{2} \log \frac{\left|z_{12}\right|^{2}\left|z_{23}\right|^{2}}{\left|z_{31}\right|^{2}} \\
& -\frac{1}{2} \log \frac{\gamma\left(\eta_{2}+\eta_{3}-\eta_{1}\right) \gamma\left(\eta_{1}+\eta_{2}-\eta_{3}\right) \gamma\left(\eta_{1}+\eta_{2}+\eta_{3}-1\right)}{\gamma\left(2 \eta_{2}\right)^{2} \gamma\left(\eta_{3}+\eta_{1}-\eta_{2}\right)}, \\
C_{3}=- & \frac{1}{2} \log \lambda-\frac{1-2 \eta_{3}}{2} \log \frac{\left|z_{23}\right|^{2}\left|z_{31}\right|^{2}}{\left|z_{12}\right|^{2}} \\
& -\frac{1}{2} \log \frac{\gamma\left(\eta_{3}+\eta_{1}-\eta_{2}\right) \gamma\left(\eta_{2}+\eta_{3}-\eta_{1}\right) \gamma\left(\eta_{1}+\eta_{2}+\eta_{3}-1\right)}{\gamma\left(2 \eta_{3}\right)^{2} \gamma\left(\eta_{1}+\eta_{2}-\eta_{3}\right)},
\end{aligned}
$$

where $\gamma(x)$ is defined by

$$
\gamma(x) \equiv \frac{\Gamma(x)}{\Gamma(1-x)}
$$

This result is exactly the same as the one derived by the conventional method in [24-26]. Therefore, using the formula (2.14), we can exactly reproduce the classical limit of the DOZZ three-point functions, first derived in [24]. Although the results themselves are already obtained, our method, which does not necessitate the explicit solutions, is quite different from the previous approaches. Accordingly, the exact match of the final results provides strong evidence for the validity and the utility of the method.

\section{Discussion and future direction}

In this paper, we have solved the semiclassical three-point functions of the Liouville field theory by utilizing the Riemann-Hilbert analysis. Instead of directly solving the classical Liouville equation, we have evaluated the Wronskians, which are the bilinears of the solutions to the auxiliary linear problem. From the monodromy relations around the punctures, we have obtained certain products of the Wronskians. By applying the method of exact WKB curves [32], we have determined the analyticity of the Wronskians and eventually 
the exact formula for the Wronskians. The success in computing the classical Liouville three-point functions strongly supports the validity of this new method.

There are several future directions worth exploring. One is to generalize our method to higher-point functions and conformal blocks. The method used in this paper is closely related to the method used in the holographic calculation of correlation functions in AdS/CFT [28-32] and in the wall-crossing phenomena of the $\mathcal{N}=2$ supersymmetric gauge theories [34]. We expect that TBA-like equations, similar to those obtained in [31, 34], will be useful to characterize those quantities. In particular, in the cases involving one irregular puncture [38, 39], we will be able to derive the famous Y-systems as in [37, 40, 41]. This will also be related to the so-called ODE/IM correspondence ${ }^{19}$ [42], a correspondence between ordinary differential equations and the functional equations in the quantum integrable models such as T-systems and Y-systems. ${ }^{20}$ Another important problem is to study the quantum three-point functions with our method. It would be particularly interesting if we can integrate our method with a novel approach to the quantum three-point functions [44], which is based on the non-commutative (quantum) spectral curve.

It is also of interest to explore the relation of our method to the four-dimensional $\mathcal{N}=2$ supersymmetric gauge theories on the omega-deformed background. In the correspondence proposed in [3], the parameter $b$ in the Liouville field theory corresponds to one of the parameters of the omega-deformation in the gauge theories. Then the semiclassical limit of the Liouville field theory corresponds to a well-known limit in the gauge theory, known as the Nekrasov-Shatashvili limit, where one of the omega-deformation parameters goes to zero. In this limit, an intriguing relation between the gauge theories and the quantum integrable models has been discovered [4-23]. This relation, together with the AGT correspondence, implies the existence of the correspondence between the semiclassical Liouville field theory and the quantum integrable models. Although one can derive various nontrivial equalities exploiting such a correspondence $[45,46]$, the origin of the correspondence is still unclear. It would be very interesting if we can get some clues for this enigmatic correspondence by investigating the relation between the aforementioned TBA-like equations and the quantum integrability in the Nekrasov-Shatashvili limit.

From a mathematical point of view, the Wronskians we have computed are connection coefficients of the hypergeometric differential equations. It might be possible to apply our method to more general differential equations and compute their connection coefficients. ${ }^{21}$ Such connection coefficients play an important role in the analysis of scattering on a blackhole background $[48,49]$ and it would be interesting to apply our method to that context. Finally, it would be important to clarify the relation of our method to the existing nonperturbative methods for quantum mechanics, such as exact WKB analysis [50, 51] and the theory of resurgence $[52,53]$.

\footnotetext{
${ }^{19}$ The generalization of the ODE/IM correspondence and its relation to the Liouville field theory is discussed also in [43] in a slightly different manner. It would be interesting to clarify the relation between two approaches.

${ }^{20}$ Such investigations are now in progress.

${ }^{21}$ For example, it would be interesting if the method of this paper can be applied to multivariate hypergeometric functions such as the Gel'fand-Kapranov-Zelevinski systems, which often appear in Mirror symmetry [47].
} 


\section{Acknowledgments}

We thank D. Gaiotto, Y. Kazama, T. Okuda, A. Sever and P. Vieira for useful discussions. In particular, S.K. thanks D. Gaiotto for kindly sharing his unpublished notes. The research of D.H. and S.K. is supported in part by JSPS Research Fellowship for Young Scientists, from the Japan Ministry of Education, Culture, Sports, Science and Technology. We would like to acknowledge the kind hospitality of Yukawa Institite for Theoretical Physics and the Kavli Institute for the Physics and Mathematics of the Universe, where part of this work was done. In addition, D.H. would like to thank the Simons Center for Geometry and Physics, and S.K. would like to thank the Perimeter Institute for Theoretical Physics, for hospitality.

Open Access. This article is distributed under the terms of the Creative Commons Attribution License (CC-BY 4.0), which permits any use, distribution and reproduction in any medium, provided the original author(s) and source are credited.

\section{References}

[1] P.H. Ginsparg and G.W. Moore, Lectures on $2-D$ gravity and $2-D$ string theory, hep-th/9304011 [INSPIRE].

[2] Y. Nakayama, Liouville field theory: A Decade after the revolution, Int. J. Mod. Phys. A 19 (2004) 2771 [hep-th/0402009] [INSPIRE].

[3] L.F. Alday, D. Gaiotto and Y. Tachikawa, Liouville Correlation Functions from Four-dimensional Gauge Theories, Lett. Math. Phys. 91 (2010) 167 [arXiv:0906.3219] [INSPIRE].

[4] N.A. Nekrasov and S.L. Shatashvili, Quantization of Integrable Systems and Four Dimensional Gauge Theories, arXiv:0908.4052 [INSPIRE].

[5] A. Mironov and A. Morozov, Nekrasov Functions and Exact Bohr-Zommerfeld Integrals, JHEP 04 (2010) 040 [arXiv: 0910.5670] [INSPIRE].

[6] A. Mironov, A. Morozov and S. Shakirov, Matrix Model Conjecture for Exact BS Periods and Nekrasov Functions, JHEP 02 (2010) 030 [arXiv:0911.5721] [INSPIRE].

[7] N. Nekrasov and E. Witten, The Omega Deformation, Branes, Integrability and Liouville Theory, JHEP 09 (2010) 092 [arXiv: 1002. 0888] [INSPIRE].

[8] J. Teschner, Quantization of the Hitchin moduli spaces, Liouville theory and the geometric Langlands correspondence I, Adv. Theor. Math. Phys. 15 (2011) 471 [arXiv:1005.2846] [INSPIRE].

[9] K. Maruyoshi and M. Taki, Deformed Prepotential, Quantum Integrable System and Liouville Field Theory, Nucl. Phys. B 841 (2010) 388 [arXiv:1006.4505] [InSPIRE].

[10] R. Poghossian, Deforming SW curve, JHEP 04 (2011) 033 [arXiv: 1006.4822] [INSPIRE].

[11] A. Marshakov, A. Mironov and A. Morozov, On AGT Relations with Surface Operator Insertion and Stationary Limit of Beta-Ensembles, J. Geom. Phys. 61 (2011) 1203 [arXiv: 1011.4491] [INSPIRE].

[12] N. Nekrasov, A. Rosly and S. Shatashvili, Darboux coordinates, Yang-Yang functional and gauge theory, Nucl. Phys. Proc. Suppl. 216 (2011) 69 [arXiv:1103.3919] [InSPIRE]. 
[13] N. Dorey, S. Lee and T.J. Hollowood, Quantization of Integrable Systems and a 2d/4d Duality, JHEP 10 (2011) 077 [arXiv:1103.5726] [INSPIRE].

[14] F. Fucito, J.F. Morales, D.R. Pacifici and R. Poghossian, Gauge theories on $\Omega$-backgrounds from non commutative Seiberg-Witten curves, JHEP 05 (2011) 098 [arXiv:1103.4495] [INSPIRE].

[15] H.-Y. Chen, N. Dorey, T.J. Hollowood and S. Lee, A New 2d/4d Duality via Integrability, JHEP 09 (2011) 040 [arXiv: 1104.3021] [INSPIRE].

[16] G. Bonelli, K. Maruyoshi and A. Tanzini, Quantum Hitchin Systems via beta-deformed Matrix Models, arXiv:1104.4016 [INSPIRE].

[17] J.-E. Bourgine, Large- $N$ limit of beta-ensembles and deformed Seiberg-Witten relations, JHEP 08 (2012) 046 [arXiv: 1206.1696] [INSPIRE].

[18] K. Bulycheva, H.-Y. Chen, A. Gorsky and P. Koroteev, BPS States in Omega Background and Integrability, JHEP 10 (2012) 116 [arXiv:1207.0460] [INSPIRE].

[19] F. Fucito, J.F. Morales and D.R. Pacifici, Deformed Seiberg-Witten Curves for ADE Quivers, JHEP 01 (2013) 091 [arXiv:1210.3580] [INSPIRE].

[20] J.-E. Bourgine, Large- $N$ techniques for Nekrasov partition functions and AGT conjecture, JHEP 05 (2013) 047 [arXiv: 1212.4972] [INSPIRE].

[21] H.-Y. Chen, P.-S. Hsin and P. Koroteev, On the Integrability of Four Dimensional $N=2$ Gauge Theories in the Omega Background, JHEP 08 (2013) 076 [arXiv:1305.5614] [INSPIRE].

[22] C. Meneghelli and G. Yang, Mayer-Cluster Expansion of Instanton Partition Functions and Thermodynamic Bethe Ansatz, arXiv:1312.4537 [INSPIRE].

[23] N. Nekrasov, V. Pestun and S. Shatashvili, Quantum geometry and quiver gauge theories, arXiv:1312.6689 [INSPIRE].

[24] A.B. Zamolodchikov and Al.B. Zamolodchikov, Structure constants and conformal bootstrap in Liouville field theory, Nucl. Phys. B 477 (1996) 577 [hep-th/9506136] [INSPIRE].

[25] L. Hadasz and Z. Jaskolski, Classical Liouville action on the sphere with three hyperbolic singularities, Nucl. Phys. B 694 (2004) 493 [hep-th/0309267] [INSPIRE].

[26] D. Harlow, J. Maltz and E. Witten, Analytic Continuation of Liouville Theory, JHEP 12 (2011) 071 [arXiv: 1108.4417] [INSPIRE].

[27] N. Seiberg, Notes on quantum Liouville theory and quantum gravity, Prog. Theor. Phys. Suppl. 102 (1990) 319 [INSPIRE].

[28] R.A. Janik and A. Wereszczynski, Correlation functions of three heavy operators: The AdS contribution, JHEP 12 (2011) 095 [arXiv:1109.6262] [INSPIRE].

[29] Y. Kazama and S. Komatsu, On holographic three point functions for GKP strings from integrability, JHEP 01 (2012) 110 [Erratum ibid. 1206 (2012) 150] [arXiv:1110.3949] [INSPIRE].

[30] Y. Kazama and S. Komatsu, Wave functions and correlation functions for GKP strings from integrability, JHEP 09 (2012) 022 [arXiv: 1205.6060] [INSPIRE].

[31] J. Caetano and J. Toledo, $\chi$-Systems for Correlation Functions, arXiv:1208.4548 [INSPIRE].

[32] Y. Kazama and S. Komatsu, Three-point functions in the SU(2) sector at strong coupling, arXiv: 1312.3727 [INSPIRE]. 
[33] E. Frenkel, Gaudin model and opers, Infinite dimensional algebras and quantum integrable systems, Prog. Math. 237 (2005) 1, Birkhäuser (2005) [math/0407524] [INSPIRE].

[34] D. Gaiotto, G.W. Moore and A. Neitzke, Wall-crossing, Hitchin Systems and the WKB Approximation, arXiv:0907.3987 [INSPIRE].

[35] H. Dorn and H.J. Otto, Two and three point functions in Liouville theory, Nucl. Phys. B 429 (1994) 375 [hep-th/9403141] [INSPIRE].

[36] J. Teschner, On the Liouville three point function, Phys. Lett. B 363 (1995) 65 [hep-th/9507109] [INSPIRE].

[37] L.F. Alday, J. Maldacena, A. Sever and P. Vieira, Y-system for Scattering Amplitudes, J. Phys. A 43 (2010) 485401 [arXiv: 1002.2459] [INSPIRE].

[38] D. Gaiotto, Asymptotically free $N=2$ theories and irregular conformal blocks, arXiv:0908.0307 [INSPIRE].

[39] D. Gaiotto and J. Teschner, Irregular singularities in Liouville theory and Argyres-Douglas type gauge theories, I, JHEP 12 (2012) 050 [arXiv: 1203.1052] [INSPIRE].

[40] L.F. Alday and J. Maldacena, Null polygonal Wilson loops and minimal surfaces in Anti-de-Sitter space, JHEP 11 (2009) 082 [arXiv:0904.0663] [INSPIRE].

[41] L.F. Alday, D. Gaiotto and J. Maldacena, Thermodynamic Bubble Ansatz, JHEP 09 (2011) 032 [arXiv:0911.4708] [INSPIRE].

[42] P. Dorey, C. Dunning and R. Tateo, The ODE/IM Correspondence, J. Phys. A 40 (2007) R205 [hep-th/0703066] [INSPIRE].

[43] V.V. Bazhanov and S.L. Lukyanov, Integrable structure of Quantum Field Theory: Classical flat connections versus quantum stationary states, arXiv:1310.4390 [INSPIRE].

[44] L. Chekhov, B. Eynard and S. Ribault, Seiberg-Witten equations and non-commutative spectral curves in Liouville theory, J. Math. Phys. 54 (2013) 022306 [arXiv:1209.3984] [INSPIRE].

[45] M. Piatek, Classical conformal blocks from TBA for the elliptic Calogero-Moser system, JHEP 06 (2011) 050 [arXiv: 1102.5403] [INSPIRE].

[46] F. Ferrari and M. Piatek, Liouville theory, $N=2$ gauge theories and accessory parameters, JHEP 05 (2012) 025 [arXiv: 1202.2149] [INSPIRE].

[47] D.A. Cox and S. Katz, Mirror symmetry and algebraic geometry, Mathematical Surveys and Monographs, vol. 68, American Mathematical Society (1999).

[48] A. Castro, J.M. Lapan, A. Maloney and M.J. Rodriguez, Black Hole Monodromy and Conformal Field Theory, Phys. Rev. D 88 (2013) 044003 [arXiv: 1303. 0759] [INSPIRE].

[49] A. Castro, J.M. Lapan, A. Maloney and M.J. Rodriguez, Black Hole Scattering from Monodromy, Class. Quant. Grav. 30 (2013) 165005 [arXiv: 1304.3781] [InSPIRE].

[50] T. Kawai and Y. Takei, Algebraic Analysis of Singular Perturbation Theory, Translations of Mathematical Monographs, vol. 227, American Mathematical Society (2005).

[51] A. Voros, The return of the quartic oscillator. The complex WKB method, Ann. Inst. Henri Poincaré A 39 (1983) 211.

[52] E. Delabaere, H. Dillinger and F. Pham, Exact semiclassical expansions for one-dimensional quantum oscillators, J. Math. Phys. 38 (1997) 6126.

[53] E. Delabaere and F. Pham, Resurgent methods in semi-classical asymptotics, Ann. Inst. Henri Poincaré A 71 (1999) 1. 